2013

\title{
Heavy flavor decay of Zgamma at CDF
}

Timothy M. Harrington-Taber

University of Iowa

Copyright 2013 Timothy M. Harrington-Taber

This dissertation is available at Iowa Research Online: http://ir.uiowa.edu/etd/4989

\section{Recommended Citation}

Harrington-Taber, Timothy M.. "Heavy flavor decay of Zgamma at CDF." PhD (Doctor of Philosophy) thesis, University of Iowa, 2013.

http://ir.uiowa.edu/etd/4989.

Follow this and additional works at: http://ir.uiowa.edu/etd

Part of the Physics Commons 


\title{
HEAVY FLAVOR DECAY OF $Z \gamma$ AT CDF
}

\author{
by \\ Timothy M. Harrington-Taber
}

A thesis submitted in partial fulfillment of the requirements for the Doctor of Philosophy degree in Physics in the Graduate College of The University of Iowa

December 2013

Thesis Supervisor: Associate Professor Jane Nachtman 
Copyright by

TIMOTHY M. HARRINGTON-TABER

2013

All Rights Reserved 
Graduate College

The University of Iowa

Iowa City, Iowa

CERTIFICATE OF APPROVAL

\section{PH.D. THESIS}

This is to certify that the Ph.D. thesis of

\section{Timothy M. Harrington-Taber}

has been approved by the Examining Committee for the thesis requirement for the Doctor of Philosophy degree in Physics at the December 2013 graduation.

Thesis Committee:

$\overline{\text { Jane Nachtman, Thesis Supervisor }}$
$\overline{\text { Yasar Onel }}$
$\overline{\text { Edwin Norbeck }}$
$\overline{\text { Wayne Polyzou }}$
$\overline{\text { Ray Culbertson }}$


This dissertation is dedicated to:

The memory of professor emeritus Edwin Norbeck, who passed away between the defense and final submission of this thesis. 


\section{ACKNOWLEDGMENTS}

I would like to acknowledge everyone who helped make this research possible.

First of all, a hearty and well-deserved thanks is directed to my advisor, Dr. Jane Nachtman, for her support, encouragement, and guidance. For helping me through the entire process, for helping me to obtain a URA Visiting Scholar award to study at Fermilab for a semester, and for all of the other invaluable assistance provided during these years, I owe her a great debt.

I would like to acknowledge Dr. Kai Yi, whose preliminary work provided the inspiration for this analysis. His Monte Carlo datasets were a key element of this analysis, and I would be deeply remiss if I did not also mention his great assistance in learning to use both the ROOT and RooFit data analysis tools.

A special thanks to the entire CDF Silicon Operations Group for allowing me the incredible opportunity to join them as a pager carrier, and thus granting me access to a great look at the CDF detector and its operations. Photographs simply do not adquately describe the experience of being in the collision hall. Also, thanks for allowing me to temporarily abandon my silicon duties in order to take shifts as an ACE in the CDF Control Room, where I had the positively unique opportunity to help run the experiment.

Thanks to my family for their tireless support and encouragement throughout all of my studies.

Finally, I would like to direct an incredible thanks to Dr. Tingjun Yang, whose assistance was absolutely indispensible for this dissertation. This dissertation owes its existence in significant part to his assistance and guidance with the analysis process, his untiring encouragement (and sometimes his unfortunately necessary lectures on why I needed to get back to work!), his experience and knowledge, and 
for his assistance in helping to write up many months of results as a formal paper. 


\begin{abstract}
Diboson production is an important and frequently measured parameter of the Standard Model. This analysis considers the previously neglected $p \bar{p} \rightarrow Z \gamma \rightarrow$ $\gamma b \bar{b}$ channel, as measured at the Collider Detector at Fermilab. Using the entire Tevatron Run II dataset, the measured result is consistent with Standard Model predictions, but the statistical error associated with this method of measurement limits the strength of this correlation.
\end{abstract}




\section{TABLE OF CONTENTS}

LIST OF TABLES . . . . . . . . . . . . . . . . . . viii

LIST OF FIGURES . . . . . . . . . . . . . . . . . . . ix

\section{CHAPTER}

1 INTRODUCTION . . . . . . . . . . . . . . . . 1

1.1 Diboson Production in $p \bar{p}$ collisions . . . . . . . . . . . . . . 1

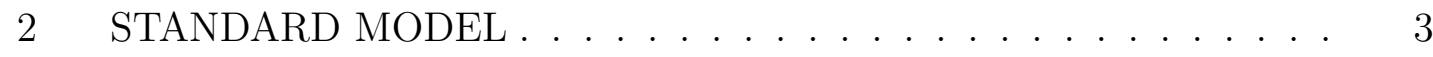

2.1 Forces . . . . . . . . . . . . . . . . . . . . 3

2.2 Fundamental Particles . . . . . . . . . . . . . . . . . . 4

2.3 Interactions with Standard Model Forces . . . . . . . . . . . . 5

2.4 Standard Model Processes at Hadron Colliders . . . . . . . . . 8

2.5 Diboson Production . . . . . . . . . . . . . . . . 9 9

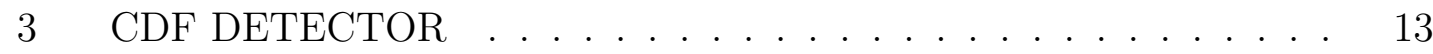

3.1 Accelerators and Elementary Particle Physics . . . . . . . . . . 13

3.2 Tevatron . . . . . . . . . . . . . . . . . 15

3.3 Collider Detector at Fermilab . . . . . . . . . . . . . . 17

4 OVERVIEW OF ANALYSIS . . . . . . . . . . . . . . 26

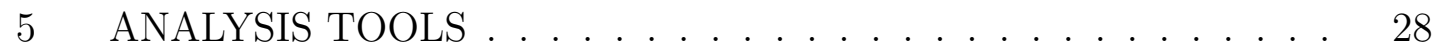

$5.1 \quad$ Artificial Neural Networks . . . . . . . . . . . . . . . . . . . . . 28

5.2 ROOT data analysis software . . . . . . . . . . . . . 31

5.3 Monte Carlo generation . . . . . . . . . . . . . 33

6 EVENT SELECTION . . . . . . . . . . . . . . 36

6.1 Datasets used . . . . . . . . . . . . . . . . . 37

6.2 Trigger efficiency . . . . . . . . . . . . . . . . . . . . 40

6.3 Photon identification . . . . . . . . . . . . . . 42

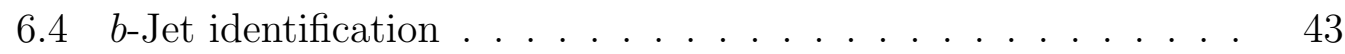

6.5 Kinematic cuts . . . . . . . . . . . . . . 44

7 DATA MODELING .................... 46

7.1 Monte Carlo datasets . . . . . . . . . . . . . . . . 46

7.2 Misidentification of photon candidates . . . . . . . . . . . . . . 47

7.3 Incorrectly tagged jets . . . . . . . . . . . . . . . . . . . 49 
8 SIGNAL YIELD CALCULATION . . . . . . . . . . . . . . 51

8.1 Neural network development . . . . . . . . . . . . . 51

8.2 Neural network fitting . . . . . . . . . . . . . . . . 54

8.3 Unfolding factor . . . . . . . . . . . . . . 56

9 ANALYSIS OF SYSTEMATIC EFFECTS . . . . . . . . . . 63

9.1 Fake photon sideband . . . . . . . . . . . . . 63

9.2 Light jet sideband . . . . . . . . . . . . . . . . . . . 65

9.3 Uncertainties in Monte Carlo reconstruction . . . . . . . . . . . 66

9.4 Uncertainties in unfolding factor . . . . . . . . . . . . . . . . 70

10 CROSS SECTION MEASUREMENT AND UNCERTAINTY . . . . 71

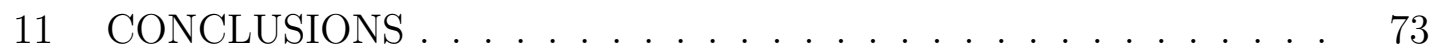

\section{APPENDIX}

SILICON DETECTOR EXPERIENCE . . . . . . . . . . . 74

A.1 Silicon Detector Technical Details . . . . . . . . . . . . . . 74

A.2 Experience with CDF Silicon Detector . . . . . . . . . . . . . 75

A.3 Technical challenges in detector construction . . . . . . . . . 75

REFERENCES ............................ 80 


\section{LIST OF TABLES}

Table

6.1 Summary of trigger requirements at different levels. . . . . . . . . . 38

6.2 Summary of cuts implemented in this analysis, excluding trigger requirements. ..................... . . . . . 45

8.1 Elements of (uncorrected) neural network fit. . . . . . . . . . 57

9.1 Expected number of events with a given number of $b$ tags for each Monte Carlo predicted data sample. . . . . . . . . . . 66

9.2 Elements of neural network fit, corrected for fake $b$ sideband. . . . . 67

10.1 Statistical uncertainties in this analysis and the resulting impact on cross section. . . . . . . . . . . . . . . .

10.2 Systematic uncertainties in this analysis and their impact upon our cross section measurement . . . . . . . . . . . . . . 


\section{LIST OF FIGURES}

Figure

2.1 Visual Representation of Standard Model . . . . . . . . . . . . . 6

2.2 Feynman diagrams for ISR and FSR events . . . . . . . . . . . . 10

2.3 Cross section for $Z \gamma$ production as a function of $E_{T}^{\gamma}$. . . . . . . . . 12

3.1 A schematic view of the Tevatron beamlines at Fermilab. . . . . . . 17

3.2 A cut-away view of CDF detector . . . . . . . . . . . . 23

6.1 Feynman diagrams for ISR and FSR events . . . . . . . . . . . 36

6.2 Mass associated with secondary vertex for jets passing inclusive $\gamma$ trigger and SVT trigger, jet $E_{T}$ between 20 and $140 \mathrm{GeV}$. . . . . . 41

6.3 SVT trigger efficiency as a function of jet $E_{T}$ in stored data. . . . . 42

7.1 Fitted $\gamma$ identification neural network output. . . . . . . . . . . 48

7.2 Fake $b$ normalization estimated using secondary vertex mass. . . . . 50

8.1 Dijet mass distribution for data and various Monte Carlo samples (Monte Carlo templates normalized to data). . . . . . . . . . . 52

8.2 Plots detailing neural network information. . . . . . . . . . . 55

8.3 Fit of neural network output, with components shown stacked on the plot. . . . . . . . . . . . . . . 56

8.4 Reconstructed $m_{j j}$ for composite fit. . . . . . . . . . . . . . . . 57

8.5 Reconstructed three-body invariant mass for composite fit. . . . . . 58

8.6 Transverse energy associated with each jet, normalized to match composite fit. . . . . . . . . . . . . . . 59

8.7 Angular separation between $\gamma$ and jets, normalized to match composite fit. ................... . . . 60 60

8.8 $\Delta \phi$ between the two jets using composite fit normalization. . . . . . 61

8.9 $\Delta \eta$ between the two jets using composite fit normalization. . . . . . 61 
8.10 Unfolding factor versus Photon $E_{T}$. . . . . . . . . . . . . . . 62

A.1 A horizontally compressed view of the CDF detector, showing $\eta$ coverage. . . . . . . . . . . . . . . . 76

A.2 CDF Run II Silicon detector, end view. . . . . . . . . . . . . 76 


\section{CHAPTER 1 \\ INTRODUCTION}

\subsection{Diboson Production in $p \bar{p}$ collisions}

Particle physics describes matter as consisting of structures of one or more fundamental particles, which interact via four fundamental forces. This model of matter and interactions is known as the Standard Model[1][2][3]. In the Standard Model of particle physics, there are many experimentally determined parameters. These parameters include the coupling of various forces and fields, the strength of the Higgs mechanism, and the imperfect symmetry between matter and antimatter[4]. The rate at which certain processes occur provides a useful measurement tool for testing these parameters. One such process is the production of multiple force mediator bosons in association with each other. Interactions which produce the carrier particles for different forces may include hints as to similarities and connections between the different particles involved[5]; one such example, production of a $Z$ boson and photon, is the topic of this thesis.

Diboson production is a frequently studied aspect of the Standard Model of particle physics, but the bulk of analyses focus on decay channels optimized for signal to background ratio, such as dimuon and dielectron decays[6][7][8][9], as well as neutrino decays[10]. Certain more highly populated channels have not been analyzed by many experiments, due to concerns that the greater background would make an analysis infeasible. One such channel which has not yet seen publication is the production of a $Z$ boson and an associated photon, where the $Z$ boson then decays into a $b$ and $\bar{b}$ quark pair. As $Z \gamma$ production is a relatively rare Standard Model process, this channel is especially sensitive to the possibility of previously unknown physics processes not included in the Standard Model.

This analysis measures the cross section of $Z+\gamma$ production where the $Z \rightarrow b \bar{b}$ 
decay occurs, so that this can be compared to Standard Model predictions. These predictions have already been tested in leptonic decay channels because hadronic colliders in general (and CDF in particular) have much lower background for leptonic processes, relative to background for quark processes, allowing for superior signal to noise ratios. Being able to check these measurements with the proposed channel will allow for improved verification of these measurements in addition to providing a possible verification of a signal for other particles which have hadronic decay modes, such as the Higgs boson.

Because this analysis only includes $b \bar{b}+\gamma$ final states, proper identification of jets associated with $b$ quark production is essential. For the CDF Run II dataset this is highly dependent upon data obtained using the silicon tracking detectors near the primary vertex to identify secondary vertices which are associated with production of heavy flavor quarks. To this end, a report on certain aspects of the CDF Run II Silicon detector is included, as well as some description of the technical support that was necessary for the continuing operation of the silicon detector. A brief summary of CDF control room operations relevant to data-taking will also be included. 


\section{CHAPTER 2 \\ STANDARD MODEL}

\subsection{Forces}

Our current understanding of physics identifies four fundamental forces, which govern how matter interacts: gravity, electromagnetism[3], the weak nuclear force[11], and the strong nuclear force[2][12]. Gravity is simultaneously the most familiar and most poorly understood force, responsible for the fall of apples to earth, as well as the earth's orbit around the sun. A satisfactory quantum description of gravity currently eludes the physics community. Electromagnetism incorporates electric phenomena due to the presence of both charge and magnetic effects, which are grounded in the motion of such charges. The weak nuclear force governs the decay of many subatomic particles, with nuclear beta decay the most familiar example. The strong nuclear forces keeps the nucleus and the constituent particles which make up the nucleus close together, overcoming their electrical repulsion. In elementary particle physics, we focus on the latter three, due to their relative strengths. An explanation of why gravity is so much weaker still awaits experimental testing, and is beyond the scope of this work. Thus, we consider the other forces together with what we consider the fundamental constituents of matter as part of the Standard Model of particle physics.

The Standard Model is dependent upon a set of parameters which govern the behavior of the different particles and forces. One such parameter is the strength of coupling with the Higgs field which gives rise to the various masses of different

particles[4]. Interactions in the Standard Model follow a number of conservation laws, some of which are familiar in composite systems. Conserved quantities include energy, linear momentum, angular momentum, and charge, as well as certain quantum numbers associated with the number of particles and anti-particles of a 
given type. Anti-particles exist for every particle, possessing equal mass and opposite electrical charge. A particle can interact with its anti-particle in a process known as annihilation, when the total mass of both particles is converted into energy, which can be used to produce a new and different pair of particles.

\subsection{Fundamental Particles}

Particles are classified as fundamental if they do not consist of smaller, more fundamental particles. Therefore, the electron is fundamental, while the proton, which consists of three quarks, is not a fundamental particle. The Standard Model divides fundamental particles into fermions and bosons, based on whether they possess intrinsic half-integer or integer spin. Spin, or intrinsic angular momentum (similar to rotational angular momentum of a macroscopic object), governs the way particles interact with other particles of the same type. Specifically, multiple integer spin bosons can exist in the same quantum state, while fermions must possess unique quantum states in accordance with the Pauli exclusion principle. Of course, not all of these particles are fundamental, so we can isolate twelve fermions (together with their anti-particle counterparts) which are not composed of other, smaller, more fundamental particles. Interactions between particles are modeled by the exchange of carrier particles, each of which is a boson and is associated with a particular force.

The fundamental fermions are classified based on whether they are capable of interacting via the strong nuclear force. Those which do interact using the strong nuclear force are known as quarks[13], while those that are not impacted by it are known as leptons. Both quarks and leptons can be divided into two more families, all consisting of three generations, based on shared properties. For quarks, these families are distinguished by charge. Those quarks with a charge equal to $2 / 3$ are one family, while those with charge $-1 / 3$ compose another. Leptons are divided 
into neutral-charge neutrinos and their associated charge -1 elementary particles (electrons, muons, and taus).

The Standard Model is depicted visually in Figure 2.1. The leftmost column contains the first generation of fermions, which are the least massive, with the heavier fermions of the second and third generation to the right. The rightmost column shows the bosons responsible for strong, electromagnetic, and weak interactions. Bosons do not follow the Pauli exclusion principle, and are the means by which Standard Model particles exchange energy and momentum in an interaction. The photon, $\gamma$, governs electromagnetic interactions. Gluons, $g$, carry the strong nuclear force. The weak nuclear force has both a charged carrier particle, $W$, and a neutral carrier particle, $Z$. Section 2.3 describes Standard Model interactions in more detail.

Due to the nature of the strong force, lone quarks are not observed, being present only in composite particles. Composite particles made up of multiple quarks are known collectively as hadrons and are separated into two categories: mesons and baryons. Mesons consist of a quark bound to an anti-quark and have integer spin. Baryons consist of three bound quarks (anti-baryons consist of three bound antiquarks) and have half integer spin. The most familiar baryons are protons and neutrons. One interesting point to note is that there is no category of anti-mesons, since swapping particles for anti-particles (and vice versa) will yield a meson (in some cases, the very same meson).

\subsection{Interactions with Standard Model Forces}

The Standard Model describes these particles as interacting via the exchange of gauge bosons, which are sometimes known as carrier particles, as they exchange energy and momentum between interacting particles as the manifestation of the 


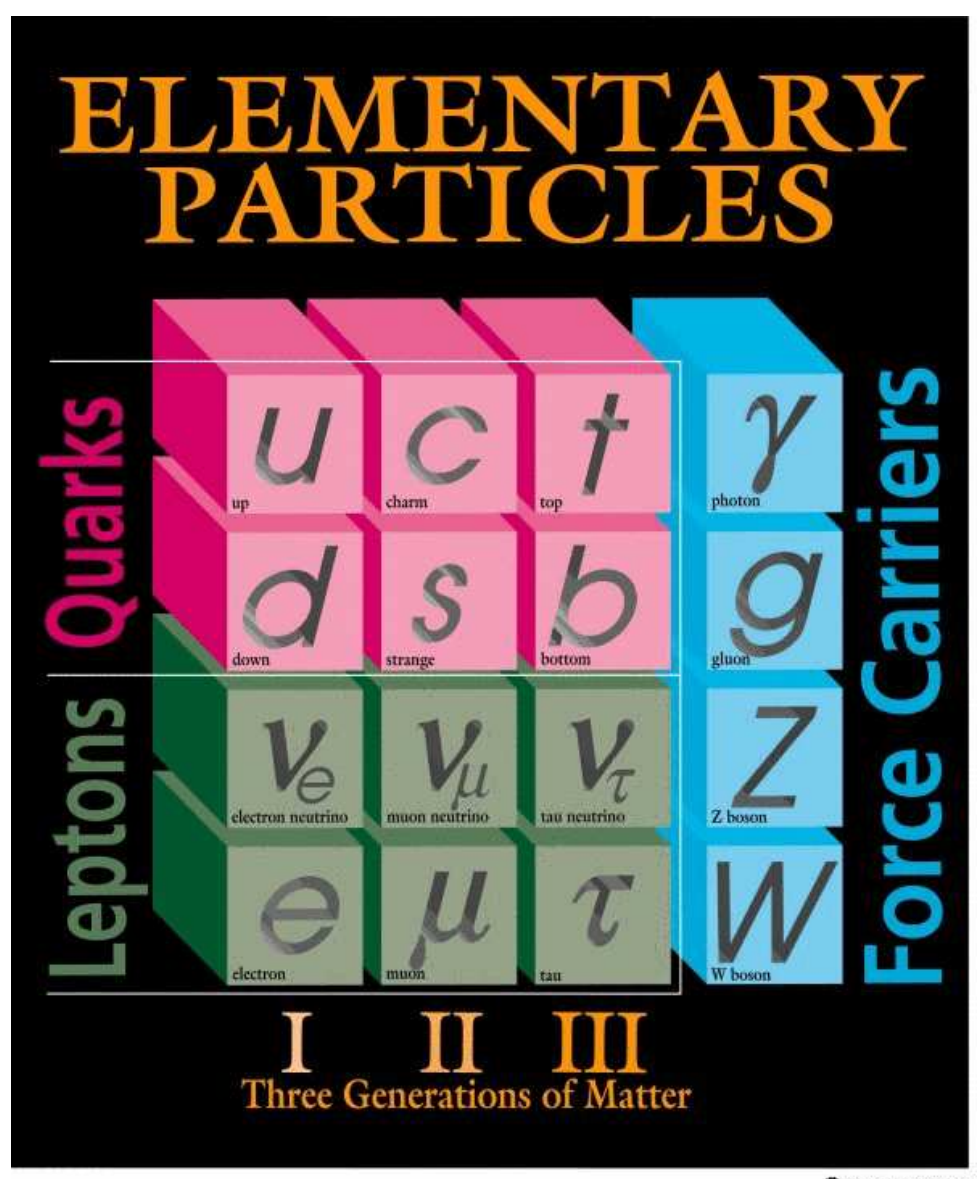

7. Fermilab 95-759

Figure 2.1: Visual Representation of Standard Model $^{1}$

force by which the particles interact. For the strong force, these particles are gluons, and the theory which concerns these interactions is known as quantum chromodynamics (QCD)[2]. The strong force obeys a unique (among elementary forces) scaling with distance known as asymptotic freedom. Thus, if various individual quarks are sufficiently separated, the gluons exchanged reach sufficient energy to produce additional quark/anti-quark pairs. Thus, lone quarks have never been observed, and their observation would greatly impact our understanding of the strong nuclear force.

\footnotetext{
${ }^{1}$ Source: Fermilab Media Services[14]
} 
For electromagnetism, the carrier particle is the familiar photon, whose massless nature permits the infinite range of electromagnetic phenomena. As the earth has a far greater mass than net electric charge, gravity is perhaps the most obvious of the fundamental forces in typical experience, and is very important at cosmological scales. What is less obvious is that most other routinely observable forces arise from electromagnetic sources, as this dominates molecular interactions which coordinate to give rise to macroscopic motion.

The weak force is responsible for more massive quarks and charged leptons decaying into less massive particles belonging to a different generation, the most familiar such process is the beta decay of atomic nuclei. In the most common beta decay process, a down quark inside a neutron decays into an up quark, changing the composite particle into a proton, with an electron and antineutrino carrying away the additional charge and energy. Particles beyond the first generation are typically only observed in cosmic rays or accelerators. Neutrinos, which interact by the weak nuclear force, and not electromagnetism (as they are electrically neutral) or the strong nuclear force, are extremely difficult to observe directly anywhere. Weak interactions can either be classified as charged-current or neutral-current, so two different carrier particles for the weak force exist: the charged $\mathrm{W}$ boson, and the neutral $Z$ boson, each of which has a mass roughly 100 times larger than that of a proton.

This analysis will focus on the $Z$ boson, which can decay in various ways, constrained by the usual conservation laws, which, since the $Z$ is a neutral particle, typically consist of a lighter particle and its anti-particle, which head off in opposite directions in the rest frame of the $Z$ boson. Based on the PDG publications[15], roughly $10 \%$ of the time, the $Z$ decays into a charged lepton/anti-lepton pair, with roughly an equal probability of each generation (as the $Z$ has a much larger mass 
than even the tau). Another $20 \%$ are classified as invisible decays, which would include all neutrino decays. The remaining $70 \%$ of the time, the $Z$ boson decays into hadrons (that is, matter consisting of quarks). Comprising $15 \%$ of the total $Z$ decays, the decay into a $b$ quark and a $\bar{b}$ anti-quark is the most common single family decay mode observed. One interesting aspect of $Z$ boson decays is that the decay into a pair of photons is forbidden by the Standard Model[5].

Of great public discussion recently is the Higgs field, which determines the observed masses of the fundamental particles[4]. According to the Standard Model, higher mass is due to a stronger interaction with the Higgs field. Since the Higgs boson (the particle associated with the Higgs field, as the photon is the particle associated with electromagnetic fields) is also electrically neutral, it can decay in some of the same ways as a $Z$ boson, making the topic of the current analysis a possible background or calibration channel for Higgs production measurements.

\subsection{Standard Model Processes at Hadron Colliders}

In the Standard Model, a proton is interpreted as a bound state of three quarks (two up, one down)[16]. In order to preserve the bound state, these quarks exchange gluons, which can also interact with other nuclear matter. At higher energies, these gluons can produce quark/anti-quark pairs of any generation permitted by their energy. To distinguish these quarks present in a proton, the nominal quark content is described as the valence quarks of the nucleon, while the less enduring quark/antiquark pairs are called sea quarks[16]. If an outside particle should happen to interact with the proton while such a pair exists, it may interact with any of these types of particles, gluons and sea quarks in addition to the valence quarks. For historical reasons, these elements of hadronic matter are known collectively as partons[16], a term which predates the quark model. At a fundamental level, interactions involve these constituent partons, and not the entire hadron. 
Hadron colliders have an important place in high-energy searches for new physics due to their interactions via all fundamental forces (gravity currently excepted as a quantum model has not been satisfactorily established). While each parton only possesses a fraction of the energy of the entire particle, which reduces the amount of energy available for making new particles, there is a greater range of parameter space available for interactions to occur. This allows for simultaneous probing of many different possible energies at the same hadron collider energy.

Although the gluons present can only interact via the strong nuclear force, the quarks present may interact via the strong nuclear force, electromagnetism, or the weak nuclear force. Thus hadron collisions, where quarks are the active elements, may involve any of the established quantum fundamental forces. The likelihood of a given force interacting is directly related to its strength, and the strong nuclear force is roughly 100 times stronger than electromagnetism and roughly $10^{6}$ times stronger than the weak nuclear force, and $10^{39}$ times stronger than gravity[17]. Thus, hadron colliders produce a large amount of strong interactions, with smaller cross sections for electroweak processes.

\subsection{Diboson Production}

One set of processes relevant to the Standard Model is the production of multiple gauge bosons in a single event. These can be used to test Standard Model predictions and to search for certain types of new physics. A noteworthy example is that many states with two bosons can be produced by the decay of a heavier particle, and the decay states of such a particle might also also match the decay states of a combination of bosons. Of especial note is the production of a $Z$ boson together with a photon. Two different Standard Model methods of generating this combination are shown in Figure 2.2. Since the $Z$ is electrically neutral, it does not interact directly with the photon, making this an intriguing potential test for new 
physics. Although the $Z$ boson does not interact directly with photons, a photon can be radiated by a quark which goes on to produce the $Z$ boson or a charged product of the $Z$ decay. When the photon is radiated by an incoming particle, it is known as Initial State Radiation (ISR), and when the photon is produced by an outgoing particle, it is called Final State Radiation (FSR).
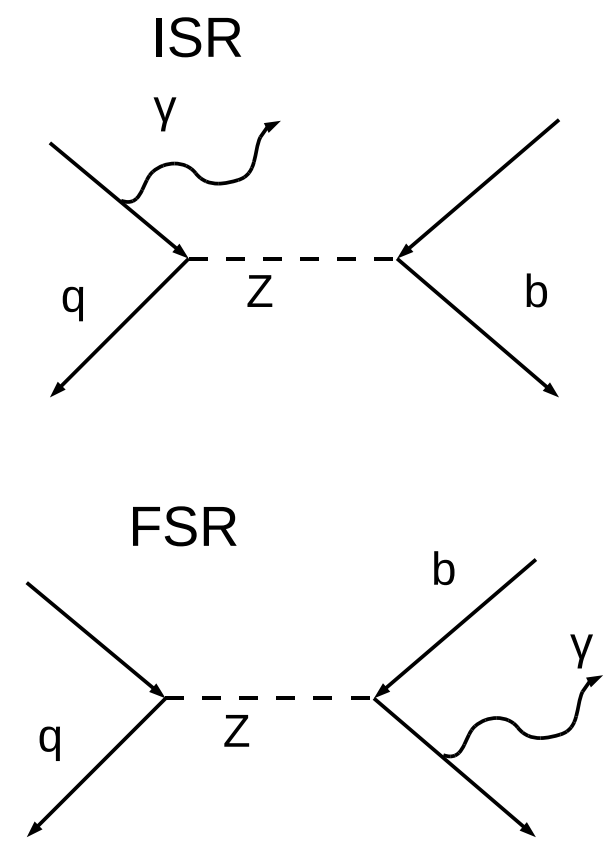

Figure 2.2: Feynman diagrams for ISR and FSR events

Initial State Radiation and Final State Radiation comprise the two established Standard Model channels for $Z \gamma$ production, but this search is sensitive to certain interactions not currently incorporated into the Standard Model[5]. One possibility it that the $Z$ boson is a composite structure of multiple charged particles. In this case, the charged component particles could produce a photon, thereby increasing the observed number of $Z \gamma$ events. Models which incorporate a composite $\mathrm{Z}$ boson 
are currently out of favor, as the observed $Z$ boson mass width is more narrow than would be expected for a composite particle[18]. Another possibility is that while there is no direct $Z \gamma$ coupling, that the three-boson couplings $Z Z \gamma$ and $Z \gamma \gamma$ might occur[6]. If either of these proposed vertices is present, the observed signal of diboson production will exceed current Standard Model predictions. Another possible enhancement of the $Z \gamma$ cross section is the possibility for a more massive neutral particle, such as the Higgs boson, to decay into a photon and a $Z$ boson. However, due to the low branching ratio of the Higgs boson decaying into a photon and $Z$ (less than one percent!), this channel is not a part of current Higgs analyses[19].

As shown in Figure 2.3, the cross section for $Z \gamma$ production decreases as $E_{T}^{\gamma}$ (transverse energy of the photon) increases, as radiation of a single photon from a charged particle (either associated with an incoming or outgoing parton, but not the $Z$ ) is less likely to produce high energy photons. Therefore, $Z \gamma$ production is highly sensitive to the chosen $E_{T}^{\gamma}$ cut, as increasing the cut can drastically reduce the signal. At the Tevatron energy level, requiring $E_{T}^{\gamma}>10 \mathrm{GeV}$, the heavy flavor final state $p \bar{p} \rightarrow Z \gamma \rightarrow b \bar{b} \gamma$ targeted by this analysis has an estimated cross section of $2.5 \mathrm{pb}[20]$. When the cut on photon energy is tightened $\left(E_{T}^{>} 15 \mathrm{GeV}\right)$, the predicted cross section drops to about $1.5 \mathrm{pb}$, a forty percent reduction. For this analysis, we require $E_{T}^{\gamma}>15 \mathrm{GeV}$, along with additional kinematic cuts, which reduce the expected cross section down to $0.35 \mathrm{pb}$. The two different cross section predictions are generated using distinct methods, making direct comparison difficult. This analysis uses the same Pythia Monte Carlo simulator used to help generate the simulated signal sample, while Reference [20] uses a method optimized specifically for diboson production[5]. 


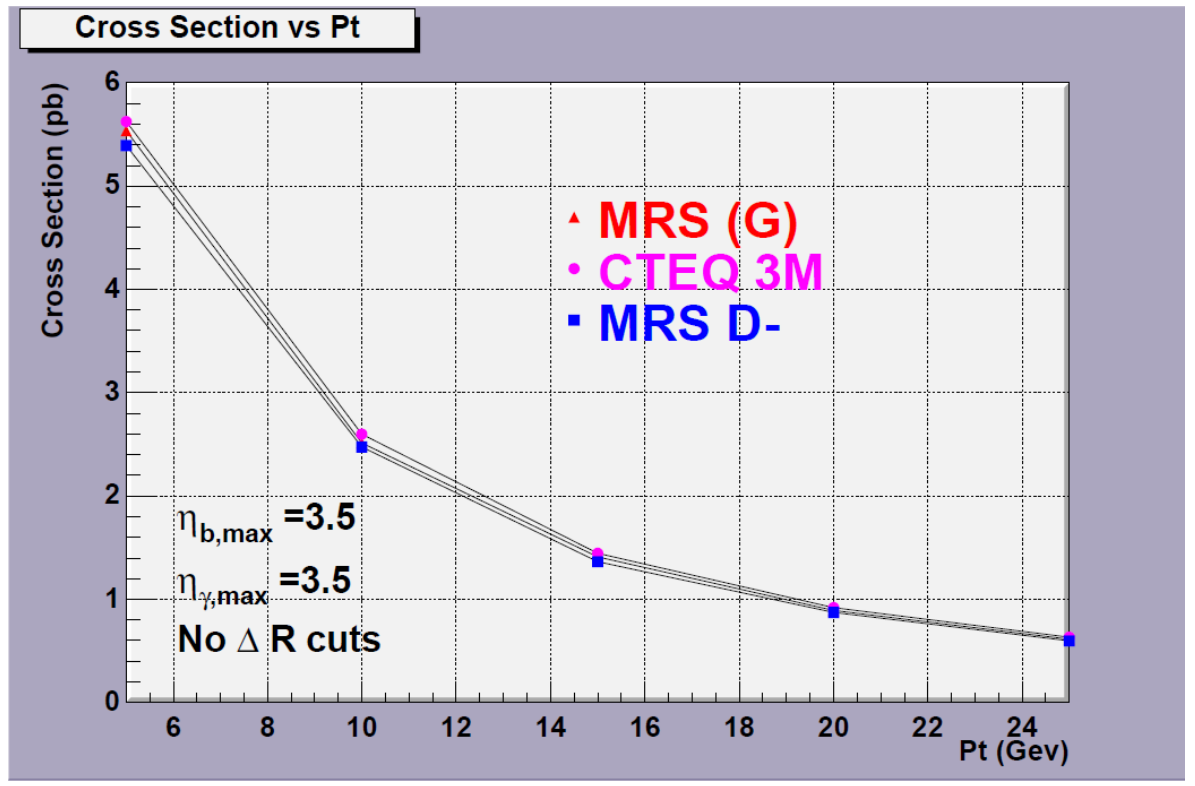

Figure 2.3: Cross section for $Z \gamma$ production as a function of $E_{T}^{\gamma}[20]$. 


\section{CHAPTER 3 \\ CDF DETECTOR}

\subsection{Accelerators and Elementary Particle Physics}

This analysis considers production of a $Z$ boson in conjunction with a photon. In order to produce $Z$ bosons, it is necessary to use very high energy particles. The current preferred method for probing Standard Model interactions is to accelerate charged particles to relativistic velocities, and then collide them either with a fixed target or with another particle of similar energy traveling in the opposite direction. Head-on collisions possess a higher energy in the center of mass reference frame than fixed target collisions, and thus are the currently preferred method of reaching the highest possible energies given current technology. Classically, two particles colliding head-on possess four times the kinetic energy of a single particle of the same energy colliding with a fixed target. Special relativity actually increases the energy gain much further than this classical value, with the equivalent kinetic energy $T_{e q}$ needed to produce the same reaction as two particles colliding with kinetic energy $T$ give by: $T_{e q}=4 T\left(1+\frac{T}{2 m c^{2}}\right)[17]$. If protons and antiprotons (mass $938 \mathrm{MeV}$ ) with kinetic energy of $980 \mathrm{GeV}$ are used, $T_{e q}$ is over 2000 times larger than $T$, a factor of 500 increase over the classical result. The technical challenge of colliding beams of particles is certainly worth it in terms of the results!

Over the years, different methods of accelerating particles to increasingly higher energies have been developed. The basic principle is to apply electric fields to a charged particle, which increases its velocity and energy. The experimental setup used to do this is known as an accelerator. In a linear geometry, a radio-frequency electrical field can be applied to a length through which the particle travels, with shielding placed so that the particle only experiences an electrical impulse in the forward direction. This method is limited in part by the ability to obtain sufficient 
land for a satisfactorily long linear accelerator.

A more compact geometry is obtainable by using magnetic fields to steer the charged particle. Using this method, a single electrical potential can be used repeatedly for the same particle, with the magnetic fields moving the particle in a curved arc so that it returns to roughly the same place. One clever method of using magnetic fields to improve accelerator performance is known as the cyclotron[21]. In a cyclotron, two regions of constant magnetic field force the particle to travel in a semi-circle, crossing and re-crossing a gap in between the two magnets, where a radio-frequency electric field accelerates the particle. This works because the particle travels at a wider radius as it accelerates, maintaining the same period of rotation as energy increases by moving to a larger radius. In a cyclotron, the particle accelerates as it moves out from the center in a spiral, eventually reaching the edge of the magnets. A variation of the cyclotron known as the synchrotron now dominates circular accelerator design. In a synchotron, only a small ring is magnetized (as compared to a large half-circle), and the magnetic field is increased to preserve a constant radius for the particle path as energy increases[22]. For a synchrotron, the physical size of the magnets is less of a design constraint than the strength of the magnets, and the amount of space needed for the appropriate radius of curvature of the accelerator.

Either leptons or hadrons may be used in such collisions, each with different characteristics. Lepton colliders typically accelerate electrons and positrons (antielectrons) in opposite directions. Many successful electron colliders have provided particle physicists with enough data to pose new questions for the next generation of accelerators. Electrons radiate energy in the form of photons when in a magnetic field, which makes it harder to reach the energy frontier with electrons in circular colliders. There are two proposed lepton colliders which would probe the 
energy frontier, both of which circumvent the difficult of electron radiation. The proposed International Linear Collider[23] does so by constructing a very long linear collider, and the Muon Collider proposes to use muons instead of electrons in the collision[24]. Lepton colliders are excellent environments for the measurement of certain energy-specific processes, because all of the beam energy goes into the interaction (this also simplifies searches for missing energy). Since leptons are fundamental particles, there are no observer elements in the interaction, and therefore no underlying interactions between observer elements.

The Large Hadron Collider at CERN (LHC) reaches the highest energies (aside from cosmic rays, which are notoriously hard to control) currently available for particle physics with data collected in 2012 at $\sqrt{s}=4 \mathrm{TeV}[25]$. Prior to the start of operations of the LHC, the highest energy collider was the (now-retired) Tevatron at Fermilab, where protons and anti-protons were collided head-on at $980 \mathrm{GeV}$ each[14]. This analysis considers the data collected at the CDF (Collider Detector at Fermilab, formerly Collider Detector Facility)[26], one of two detectors present at the Tevatron.

\subsection{Tevatron}

Although eventually surpassed by the Large Hadron Collider at CERN, the Tevatron accelerator at Fermi National Accelerator Laboratory was for many years the highest energy collider in the world. Over the course of 25 years, the Tevatron produced proton/anti-proton collisions using magnetic fields to direct the two counter-propagating beams, with the beams focused to greatly increase collision rate at the site of the two detectors along its main ring, $\mathrm{CDF}[26][27]$ and $\mathrm{D} 0[28]$. These two detectors provided data used in many analyses, most famously the discovery of the top quark[29].

The Tevatron is a multi-stage accelerator, with the final stage consisting of 
the main collider ring, where the collisions occur at fixed points (the center of the CDF and D0 detectors). Protons are generated by ionizing hydrogen gas, and selected based on charge. Anti-protons are generated from protons colliding at high energy with a fixed target. Outgoing anti-protons are separated based on their unique charge/mass ratio, and circulated through a recycler ring by external magnetic fields. This preserves the valuable anti-protons from their destruction by interaction with matter, which has a rather high proton population. The primary beamlines for the Tevatron collider, together with beams of particles destined for fixed-target experiments, are superimposed upon an aerial photograph of Fermilab in Figure 3.1.

In order to reach the $1.96 \mathrm{TeV} E_{c m}$ desired, each beam is accelerated to 980 $\mathrm{GeV}$. This is done through several stages, each of which increases the proton energy. Freshly ionized protons are passed through a Cockcroft-Walton[30] preaccelerator, which increases their energy to $750 \mathrm{keV}$. Next, these are exposed to a radiofrequency linear accelerator (shielded so as to only present one voltage polarity to the protons), which boosts energy to $400 \mathrm{MeV}$. A booster synchrotron increases the energy of these $400 \mathrm{MeV}$ protons up to $8 \mathrm{GeV}$, at which energy the protons pass into the Main Injector. The Main Injector is an elliptical synchrotron which increases energy of protons to $150 \mathrm{GeV}$ for injection into the main Tevatron ring. The Main Injector also (separately) raises the anti-proton energy to $150 \mathrm{GeV}$. At $150 \mathrm{GeV}$, both protons and anti-protons are loaded into the main Tevatron collider ring, a synchrotron which brings each beam to $980 \mathrm{GeV}$, and preserves that orbit while the beams slowly depleted each other via collisions (and while more anti-protons were being collected for the next set of collisions). Superconducting magnets allow the Tevatron to produce and maintain the strong magnetic fields necessary for the operational energy. Additional information is available through the Fermilab website, 
http://www.fnal.gov/pub/science/accelerator/.

\section{FERMILAB'S ACCELERATOR CHAIN}

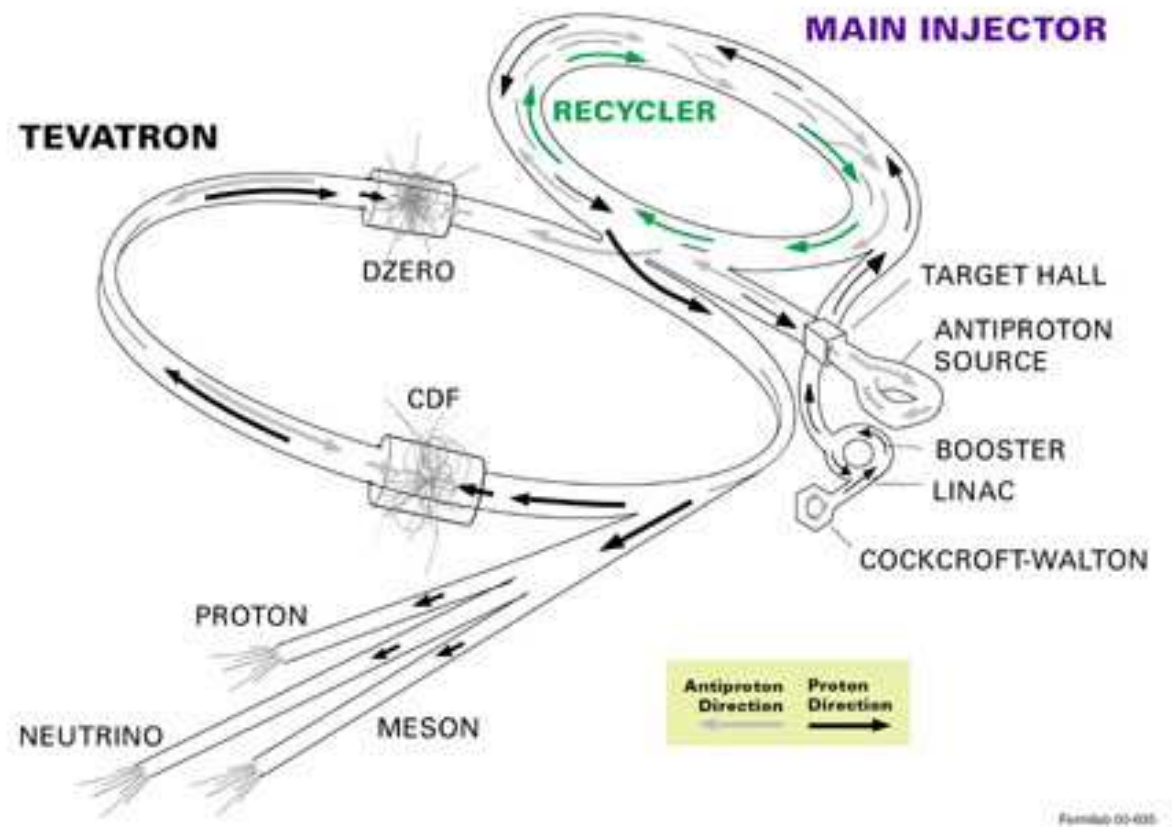

Figure 3.1: A schematic view of the Tevatron beamlines at Fermilab. ${ }^{1}$

\subsection{Collider Detector at Fermilab}

In order to study high-energy particle interactions, specialized detectors are built near the interaction region in order to observe the outgoing particles and obtain the information necessary for analysis. Typically, some method of tracking

\footnotetext{
${ }^{1}$ Source: Fermilab Media Services[14]
} 
charged particles is used near the interaction region, with a magnetic field applied to gain momentum information about charged particles, and calorimeter detectors are placed outside of the tracking region to bring particles to a stop in order toestimate energy.

Because each particle in a collider detector has substantial energy due to its motion in the direction of the beam's travel, energy and momentum are frequently calculated only in the plane orthogonal to the direction of motion. That is, only momentum due to motion perpendicular to the beamline is included in these calculations, and any energy due to motion in the direction of the beam is ignored. When used in this manner, these are called transverse momentum $\left(p_{T}\right)$ and transverse energy $\left(E_{T}\right)$. As precise measurement of outgoing momentum along the beam axis is very difficult, these quantities are used for this analysis.

In order to designate direction inside the detector, a polar set of coordinates are defined. At CDF, the angle $\theta$ is the angle made relative to the direction of motion of the incoming protons. The angle $\phi$ is the angle relative to the beam axis. Rather than use $\theta$, a related quantity, known as the pseudorapidity is defined as $\eta=-\ln [\tan (\theta / 2)]$. A particle traveling exactly perpendicular to the beampipe will have $\eta=0$, and the magnitude of the pseudorapidity increases at angles closer to the beampipe[31]. Pseudorapidity has the convenient property that differences in $\eta$ are invariant under boosts along the beam axis. This means that the difference in $\eta$ for particles in an event is the same, even if the net boost of the interacting particles changes.

This analysis measures events where a photon and a $Z$ boson are produced, where the $Z$ decays into a $b$ quark and its anti-quark. Since the $Z$ is much more massive than two $b$ quarks, this quark pair has high energy. When a quark pair is produced at high energy, it quickly generates a number of hadrons out of the 
quark/anti-quark pairs produced by the separation between the original quark/antiquark pair. These hadrons tend to follow similar trajectories, centered around the direction of motion of the original quark. This group of hadrons is called a jet by the particle physics community, and since a single interaction at Tevatron energies is capable of producing hundreds of particles[14], considering a jet as a composite whole can greatly simplify some analyses. Production of a high energy quark is observed in a detector as a narrow jet of particles (mostly hadrons). Since $b$ quarks are more massive than their smaller fellow quarks, they decay relatively quickly. Because the $b$ quarks are generated at relativistic energies, they are boosted, and travel a measurable distance from the interaction region before decaying. This decay produces a secondary interaction point which appears to the detector as the jet originating at this secondary vertex. Therefore, it is necessary to identify these secondary vertices in order to distinguish $b$ jets from lighter flavor jets. At CDF, this is done using tracking information obtained from silicon strip detectors near the interaction point[31]. A more thorough description of the silicon detector can be found in Appendix A, but the general function of the CDF detector is described here. Thorough descriptions of all systems are detailed in the Technical Design Report for the CDF detector[26].

The silicon subdetector system at CDF consists of three different systems, each of which consists of silicon strip trackers to provide the most thorough information possible as to the paths taken by charged particles near the interaction point. The innermost system, known as Layer 00, rests directly on the beampipe, a few short centimeters from the interaction point. This system of single-sided silicon strips improves resolution of tracks near the primary vertex, but requires more stable beam conditions in order to operate safely (as compared to the D0 detector also present at the Tevatron). The second system in the silicon detector is called SVX, 
which consists of 6 layers of double-sided silicon strips. The outermost system is the set of Intermediate Silicon layers (ISL), which provide more tracking via silicon strip detectors, while still resting inside the other parts of the detector. The ISL subdetector consists of five double-sided silicon strips, each mounted in a staggered pattern to provide additional tracking between SVX and the rest of the detector. Depending on the angle at which a particle travels with respect to the beam pipe, it encounters one or two of these ISL strips. These three systems are referred to collectively as SVX II, as they constitute a clear upgrade over the silicon tracking present in the first Tevatron run[31].

Outside of the silicon detector, additional tracking information (highly useful for identifying more stable charged particles that reach the calorimetry systems) is provided by a gas-filled chamber crossed by many wires known as the Central Outer Tracker (COT). Similarly to how charged particles ionize silicon and cause current to flow in the silicon tracking systems, the gas (a mixture of argon and ethane) is ionized by charged particles moving through the gas, and these ions drift towards the wires strung throughout the chamber. The charge picked up by these wires is used to determine the paths of particles present in the decay. As the entire tracking region is inside a superconducting solenoid, which produces a magnetic field of 1.4 Tesla[26][27], the radius of curvature of these tracks allows reconstruction of the charge/energy ratio (as particles generated are typically relativistic, the charge/mass ratio is more difficult to extract).

It is important to note that both the silicon detector and COT could only detect charged particles. Neutral particles escape tracking and their presence must either have be inferred from changes in the tracks of charged particles, or be observed by the energy that they deposited in matter. One noteworthy aspect of tracking chamber design is to limit the amount of energy that particles lose while passing 
through the tracking region. This is due not only to the fact that losing energy causes a particle to also lose momentum (strictly speaking, this energy and momentum is transferred into the medium of the tracking chamber, in accordance with energy conservation), but it also reduces the accuracy of calorimeter measurements of particle energy and alters particle direction.

Outside of the tracking region, the CDF detector has both electromagnetic and hadronic calorimeters[26]. These systems consist of layers of scintillator material sandwiched between layers of iron or lead, respectively, designed to interact with the outgoing particles, creating a shower of lower-energy particles, which continues until the particles are of sufficiently low energy that they stop entirely. A scintillator is a transparent material that produces light when charged particles pass through it, so the amount of light detected by a photodetector in a given layer is related to the number of particles traversing that layer. As there is a certain maximum energy at which the particles can be stopped in a given layer of a particular metal, it is possible to reconstruct the total energy of a given incident particle based on the size of the shower present in the calorimeter. Because two different materials are used, some particles will stop in the metal and not reach the scintillator, where they are detected by the electronics. Therefore, the total amount of energy is estimated based on the sample of charged particles which are observed passing through the scintillator.

The electromagnetic calorimeter is optimized to stop electrons and photons, consisting of lead interspersed with scintillator material[26], while allowing hadrons to pass through with relatively little loss of energy. The hadronic calorimeter has thicker iron layers (to increase energy loss from hadrons), and is used to estimate as best as possible the energy of incident baryons and mesons. The electromagnetic calorimeter is designed to compose 19 interaction lengths for electrons and 
one interaction length for hadrons, while the hadronic calorimeter supplies another 4.5 interaction lengths for hadrons[26]. Individual calorimeter elements, each representing a specific solid angle region of the detector, are known as towers. These towers are grouped into wedges of 15 degrees each, split again between east and west sides. Each wedge is approximately sixteen tons. Although not used in this analysis, an additional layer of muon detectors are outside of the calorimeters, as muons do not interact strongly with the calorimeter material (the rationale is that any charged particle still present at that point is most likely a muon)[26]. As muon events are relatively rare, they are frequently used to reduce background for many analyses, including most diboson production analyses. In order to confirm appropriate functioning and to correct for drift in behavior over time, standard tests are run on the detector systems when data is not being actively collected in order to calibrate the systems.

Due to the sheer amount of data relevant to every event, and the limitations of the data processing software and hardware available when CDF Run II was designed, only several hundred events per second are retained for analysis. As bunches of protons and antiprotons cross paths in the middle of the detector 1.7 million times a second, with multiple interactions possible in every beam crossing (the instrumentation is designed expecting three interactions in a typical crossing), it is obvious that significant pruning must have occurred before events could be saved for analysis. In order to determine which events are preserved for analysis, the CDF detector uses a three level trigger system, which was chosen to attempt to maximize the amount of data deemed most interesting by the particle physics community[32].

To further optimize performance, the trigger system is broken down into three levels, each one more complex than the previous, and which reduces the events 


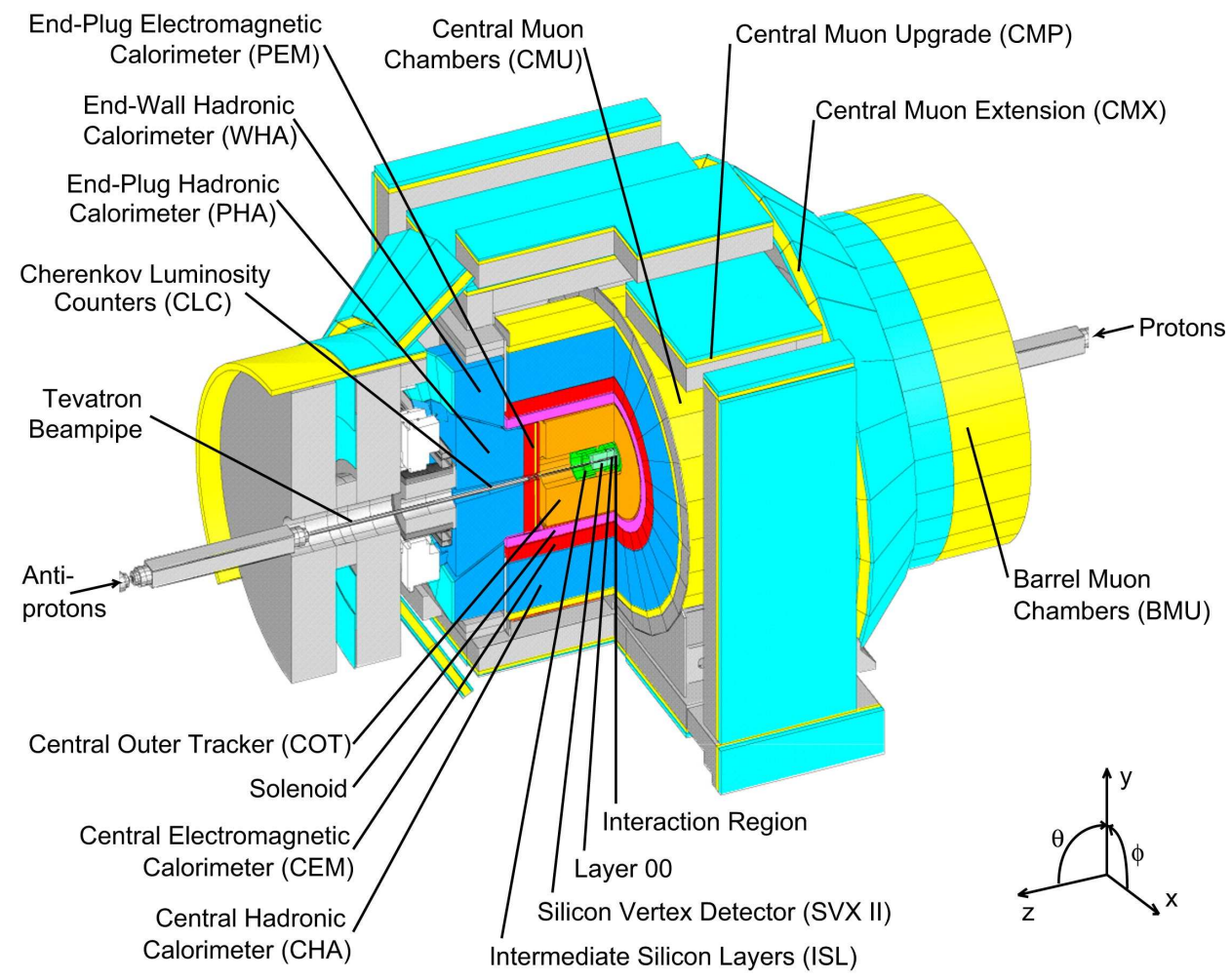

Figure 3.2: A cut-away view of the CDF detector ${ }^{2}$

retained for further analysis. Custom hardware and firmware, specific to CDF, implement this trigger according to a compromise between calculation speed and the ability to incorporate as many interesting physics processes as possible. As many different elements of the detector are present in this process, when data is not being actively collected (typically when a new quantity of protons and antiprotons are being loaded into the collider ring), the different detector elements are

\footnotetext{
${ }^{2}$ Source: Fermilab Media Services[14]
} 
re-calibrated, and these calibrations checked for accuracy.

The first level selects roughly 25,000 events per second out of 1.7 million bunch crossings, based on the fastest readout information, and temporarily stored data from those events for further selection. Level 1, as this selection level is known, only considers the results of individual regions of particular systems, for maximum speed. The second level reduces these events to about 900 per second, obtaining information from each element of the detector. One noteworthy element of the Level 2 trigger is the ability to incorporate data from the SVX II system into the decision-making process[26]. This trigger, based on the silicon tracking system, is known at the Silicon Vertex Trigger (SVT), and is the first major use of silicon data collection in the trigger process for a particle physics experiment. The third and final level takes these events and reconstructs the event in question (the trajectories of charged particles and the energy associated with each particle), and preserves 200 events per second for later analysis[32].

Because CDF produces many different types of events interesting to the particle physics community, the trigger process takes into account a number of different sets of observables deemed interesting to the collaboration. Each of these is helpfully referred to as its own trigger, and it is the collection of the various triggers which is used by the hardware and software to determine which events are retained for further analysis. Due to the constraints of data-taking, each of these specific triggers can be set to thin out events which meet its criteria, so that the detector is able to maintain a roughly constant rate of event retention, even as the interaction rate declines. To avoid distorting analysis, the events which pass a specific trigger are flagged as such, and are incorporated into a dataset. This dataset contains all events which passed the associated trigger and were preserved for analysis.

At this point, detector data is fed into a farm of computers, working in parallel, 
in an attempt to, as best as possible, reconstruct the paths and energy of individual particles present in the event. This process compares track information and calorimeter information to identify the various particles produced in a given interaction, and the energy possessed by each particle. With this particle identification information, the data is considered ready for analysis. 


\section{CHAPTER 4 \\ OVERVIEW OF ANALYSIS}

This analysis measures the cross section of the production of a $Z$ boson associated with a photon, where the $Z$ decays into two heavy flavor jets, based on gamma $+b \bar{b}$ data collected at Fermilab National Accelerator Laboratory throughout CDF Run II, corresponding to an integrated luminosity of $9136 \pm 548 \mathrm{pb}^{-1}$. At hadron colliders, due to the relative strengths of the fundamental forces, strong interactions dominate. Therefore, many QCD processes will produce event signatures to $\gamma+Z$ production, where the $Z$ decays hadronically. For this reason, hadronic end states of electroweak processes are infrequently studied at hadron colliders, due to the large presence of QCD processes with the same outgoing particles[5]. A search for $\gamma+Z$ production was made at CDF, including all possible hadronic decay modes, but did not obtain a conclusive result[33], due to lack of sufficient separation between signal and background for the statistics available. This measurement supports the Standard Model prediction for diboson production, as previously observed in leptonic decay channels[8].

This analysis aims to improve upon the previous result for hadronic decays by incorporating the entire CDF Run II dataset and selecting only heavy flavor $Z$ decays. To reduce the background ratio, this analysis considers only the highest mass hadronic mode $(b \bar{b})$, which also has a slightly higher branching fraction than other similar hadronic decays. In order to do this, it is necessary to have an effective method of distinguishing $b$ quarks from lighter quarks.

Due to significant kinematic overlap between signal and irreducible background (that is, events not involving a $Z$ boson that still had the same $\gamma+b \bar{b}$ signature), this analysis uses a neural network to distinguish between these two types of events, and fits the neural network output to templates generated by the 
Monte Carlo samples which simulate the two types of events. A further discussion of the nature of these samples and the tools used to analyze them can be found in Chapters 5, 6, and 7 . 


\section{CHAPTER 5}

\section{ANALYSIS TOOLS}

This analysis incorporates several specialized tools in order to accurately identify $\gamma+Z$ events, model signal and background behavior, calculate uncertainty, and manage the data analysis process. Because artificial neural networks are critical to this analysis, a description of the structure and function is provided. Due to the complexity of the CDF data, it is not possible to review events manually. In order to efficiently analyze particle physics data, physicists write computer scripts to manage the particular steps of the analysis. This analysis uses a $\mathrm{C}++$ based data analysis software package known as ROOT. In order to compare data with theoretical expectations, a Monte Carlo process is used to simulate events according to theoretical predictions. This chapter describes the nature and use of each of these tools, as used in this analysis.

\subsection{Artificial Neural Networks}

The term neural network is derived from the word neuron, and is used to describe both natural and artificial systems that function in a similar manner, based on how the brain functions[34]. One attempt to model mental processes in animals is to connect electrodes to various neurons, and try to determine correlations in neural activity in specific neurons.

Artificial neural networks attempt to replicate this process by setting up a network of nodes, with connections between nodes adjusted to possess the desired behavior. This analysis uses a particular type known as a multilayer perceptron, which is used here to distinguish between two data samples based on different values for physical measurements (and simulated equivalents) obtained in the experiment.

A multilayer perceptron takes as its inputs two samples of simulated collisions reconstructed using a simulation of the CDF detector (the simulation process is 
described in Section 5.3), each tagged, and attempts to generate a non-linear relation that yields a numerical value between 0 and 1, with values closer to 1 signifying events more similar to those described as signal and values closer to 0 being more typical of background. A multilayer perceptron consists of a set of input nodes (the different variables to be used to discriminate between the data sets), one of more layers of hidden nodes, each of which depends mathematically on the previous layer (nodes in the first hidden layer are functions of the input nodes), and one output node, which is a function of the nodes in the last hidden layer. The functions by which each layer depends on the previous layer are adjusted iteratively to improve the separation between signal and background in the output.

Determining the most appropriate neural network for a particular analysis is not trivial, and is somewhat subjective[35]. In principle, the quality of a neural network is determined by the precision of its ability to distinguish between the different types of events. Therefore, in order to absolutely determine which neural network is preferable, the most definitive method would be to complete the entire analysis for each network, and to use the network that yielded the smallest error. However, since the neural network output depends upon a variety of systematic effects, this process is non-trivial, and instead neural networks were evaluated based on the desirability of the output template shapes for signal and primary background. An idealized artificial neural network would output a value near 0 for all background events and a value near 1 for all signal events. The photon identification artificial neural network used to enforce the purity of the $\gamma$ signature in the data very nearly approximates this behavior (though there are small numbers of events present in the intermediate region, and a small number of background events that are present in the signal region), while the multilayer perceptron developed for this analysis falls short of this ideal. 
In order to determine which neural network architecture should be used for this analysis, it was necessary to make a judgment as to the value of various neural networks. One important caveat is that while increasing the number of iterations used to generate the network will improve the separation of the training samples, it may have several unwanted features. Most salient is the possibility of "overtraining," the situation where the neural network becomes overly dependent upon specific aspects of the particular training samples used, rather than on the general behavior of the overall distribution of actual events. In order to combat this tendency, it is typical to set aside a number of events to be used to test the performance of the network, while other events are used specifically to train the network. Thus, with each training iteration, it is possible to numerically compute the separation in both the training and test samples. After a certain point, the performance of the network starts to become less effective for the test sample while still improving the training sample performance. This phenomenon is the heart of over-training.

There is no clear way to determine from first principles the best architecture for the neural network itself. A judgment must be made based on the shape of the distribution of neural network outputs for signal and background events (ideally, this should encompass both test and training samples[35]). It is highly desirable that the output distribution for signal events forms a clear peak with a value relatively close to 1 . This peak should be relatively smooth, and not have too much fluctuation (which would suggest too small of a training sample). Likewise, it is undesirable for the background distribution to appear similar to the signal distribution, as it then becomes difficult to distinguish the two. Furthermore, it is quite valuable for the background distribution to be smooth in the region of peak of the signal distribution. This can be a flat distribution, or it can be a smoothly declining distribution. In both cases, it is quite desirable to have limited fluctuations and peaks outside the 
signal region, as this can lead to fitting based on those fluctuations, which are not necessarily strongly descriptive of the idealized distribution. An exception can be made if there is minimal background present in the signal peak, of course, but in cases where there is significant overlap between the two sample distributions, the smoother the two signals are, the more desirable the neural network.

These are, of course, means to the overall objective of developing a neural network, which is to have a reliable tool for distinguishing between signal and background. To this end, neural networks are judged by the template shapes that they produce. The primary objective is to have a clear difference between the two distributions in the signal peak region, while having the distributions sufficiently clear to ensure that the fitting algorithm depends most strongly on behavior in the peak region.

This analysis uses two distinct neural networks. The first neural network is used for identification of photon candidates, and is a standard network developed for such purposes at CDF[36]. The second neural network was developed in the course of this analysis and trained to distinguish between signal and background events. The development and implementation of this neural network was the primary avenue for this analysis.

\subsection{ROOT data analysis software}

The number of events, and the amount of information relevant to each event, effectively require the use of computer scripts in order to adequately process, evaluate, and display data relevant to the analysis in any reasonable length of time. To facilitate this process, the particle physics community has developed some specialized coding tools for use in particle physics analysis and research. This analysis uses the ROOT system of $\mathrm{C}++$ libraries, which is the current standard for particle physics research[37]. Originally developed in 1995, and an official product of CERN 
since 2002, ROOT is an object-oriented framework for data analysis, with a large number of predefined objects and associated functions, optimized for the needs of the particle physics community. The layered class hierarchy of ROOT incorporates roughly 1200 classes, contained in 60 libraries, which are divided into 19 main categories. These libraries allow for many predetermined mathematical operations, histogram manipulation, graphical presentation of data, and much more. Unless otherwise noted, all steps of this analysis were performed using ROOT libraries and commands.

While ROOT commands can be performed in command line format, it is far more typical (and easier!) to write dedicated scripts, which are then executed in command line form by the ROOT software. These scripts are written in standard $\mathrm{C}++$ syntax, while using the additional ROOT objects and functions as appropriate for the analysis. One notable attribute is the ability to import data in a standard format (known as an n-tuple) for use in a particular analysis, without changing the original files. This allows for the construction of appropriate histograms, plots, and other objects relevant to the analysis, while having previously selected only the relevant events from a larger data sample. A discussion of the data selection process is included in Chapter 6.

Since this analysis is heavily dependent upon performing complex fits, the default ROOT architecture was considered a bit unwieldy for some of the desired functionality. In order to obtain greater ability to analyze data, this analysis uses the RooFit [38] package for ROOT to perform most fitting functions. Originally developed for use by the BaBaR collaboration at Stanford Linear Accelerator Laboratory, RooFit is an additional set of classes for ROOT, designed for more flexible and intuitive fitting. RooFit defines a wide range of predetermined functions and provides support for combining multiple such functions as required. One especially 
useful tool is the ability to describe certain functions (or histograms!) as probability distribution functions for use in fitting data samples. By default, RooFit is a log-likelihood fitting algorithm, and is capable of fitting both one-dimensional and two-dimensional distributions of data, as well as providing support for graphical display of such distributions. For this analysis, the histograms derived directly from the data sample are fitted to histograms which are considered to model the various components present in the data sample. As RooFit provides a great number of tools for generating distribution functions and fitting data to these functions, it is quite useful for this analysis.

\subsection{Monte Carlo generation}

In order to adequately distinguish between different types of events present in data collected at CDF, it is necessary to have the best possible understanding of how these events differ from each other. Due to the complexities and uncertainties involved, deriving this from Standard Model principles directly is not feasible. Instead, standard procedure is to use a set of computer algorithms to generate a simulated set of events that follow Standard Model behavior (or expected behavior based on physics not in the Standard Model, if that is relevant to the analysis), with actual values for parameters chosen probabilistically based on current best understanding of the process in question. This process of running repeated simulations based on probability to obtain a data sample is known as Monte Carlo generation.

Monte Carlo generation for particle physics requires several steps. Initially, the process to be modeled is defined at the most basic relevant level. This method, known as parton-level generation, deals only with fundamental Standard Model particles (quarks, gluons, leptons, and force-carrying gauge bosons). In this model, protons are simulated as a mixture of quarks, anti-quarks and gluons, with the probability of a given type of particle being involved determined by empirically 
based parton distribution functions (uncertainty in how to best model these provides some systematic uncertainty, detailed later). This analysis used the MadGraph[39] software to simulate these parton-level interactions.

As detailed in Chapter 2, individual partons (including quarks) are not necessarily the same particles that interact with the detector. To this end, it is necessary to simulate the formation of jets as well as any decays of unstable particles. This is also determined using a Monte Carlo process (in this case, a separate one), and is described as showering. This shower of simulated particles is then sent through a simulation of the CDF detector, and the response of the CDF detector is simulated to obtain simulated data of the relevant process as it would be observed and recorded by the detector. This analysis uses the Pythia[40] Monte Carlo generator to handle showering effects and a standardized GEANT simulation of the CDF detector[41].

In order to accurately reflect detector behavior, the event reconstruction process of the CDF detector must be modeled as well as the individual detector elements. This means that the Monte Carlo process must simulate detector status, response, and reconstruction algorithms in order to most accurately simulate the data collected by the detector.

Monte Carlo generation is a powerful tool for generating a sample of data based on our best knowledge of how the events in question are observed in the detector, as well as providing an estimate as to how frequently such events occur. In cases where the data generated via these Monte Carlo methods is similar to the data observed in the detector, this strongly suggests accurate modeling. A discrepancy between Monte Carlo and observed data can have one of two causes: an incorrect application of the simulation process, or simulation software which does not incorporate all of the processes present in the data. This latter case is indicative of a certain degree 
of either new physics or a need to adapt existing physics models. Such events are quite exciting, but not present in the present analysis. 


\section{CHAPTER 6 \\ EVENT SELECTION}

Because this analysis considers production of a $Z$ boson in conjunction with a photon, and requires that the $Z$ boson decay into a $b$ quark and $\bar{b}$ antiquark, this analysis considers only events reconstructed as having at least one photon candidate, and at least 2 b-tagged jets. There are two methods of $Z \gamma$ production, distinguished based on whether the $\gamma$ is associated with an incoming parton or an outgoing quark. We designate $p \bar{p} \rightarrow Z \gamma \rightarrow b \bar{b}$ as Initial State Radiation (ISR), as the photon production is prior to the decay of the $Z$ boson, while $p \bar{p} \rightarrow Z \rightarrow \gamma b \bar{b}$ is labeled as Final State Radiation (FSR). (Feynman diagrams for these processes are shown in Figure 6.1). We isolate $p \bar{p} \rightarrow \gamma b \bar{b}$ events in our data sample, and attempt to determine how many of these result from $Z \gamma$ production, and how many are from other sources (most notably QCD interactions).
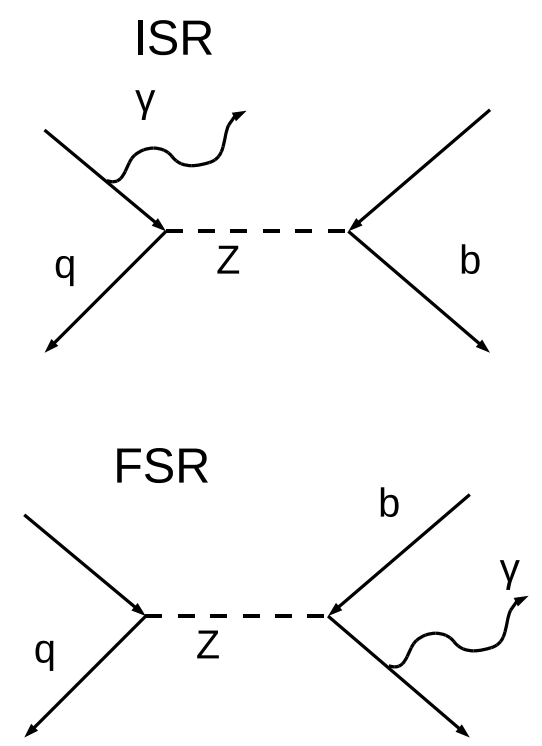

Figure 6.1: Feynman diagrams for ISR and FSR events 


\subsection{Datasets used}

Since the CDF detector separates out events based on the trigger which determined that the particular event should be stored, analysis begins by selecting the most appropriate trigger dataset. Because this analysis looks for a photon as well as heavy-flavor jets, the $\gamma+\mathrm{SVT}$ trigger is most appropriate for this analysis. This dataset (known internally as $c p h b$ ) consists of events with an isolated photon candidate together with a secondary decay vertex near the primary interaction point. The full trigger requirements are specified in Table 6.1.

The Level 1 cuts require that a specific calorimeter tower record at least 12 $\mathrm{GeV}$ of $E_{T}$, with this energy strongly concentrated in the electromagnetic calorimeter. At Level 2, these cuts are widened to include the local cluster of detector electronics, while enforcing that the event is central and that there is minimal energy is the area near the photon. At this point, the SVT cuts are applied: specifically, there must be at least one SVT track, with a certain minimum probability of the reconstructed track being valid (as determined by the $\chi^{2}$ value associated with the fit), and with at least the minimum transverse momentum and an impact parameter between $0.12 \mathrm{~mm}$ and $1.00 \mathrm{~mm}$ from the primary interaction point, while still being distinct from the primary vertex. The Level 3 cuts require that the COT offline tracking match the SVT trigger tracking, and that the distribution of energy in the electromagnetic calorimeter be deemed sufficiently associated with the track. Note that Level 1 cuts are entirely based on the photon candidate, and that jet requirements are not added until Levels 2 and 3.

In order to test the efficiency of the SVT portion of this trigger, a comparison is made to the inclusive photon dataset (dataset cph1), which does not require the SVT trigger, but requires $E_{T}^{\gamma}>25 \mathrm{GeV}$, a significantly tighter cut. This will be 
Table 6.1: Summary of trigger requirements at different levels.

Level 1

Trigger Tower $E_{T}$

$>12 \mathrm{GeV}$

Trigger Tower $E_{H A D} / E_{E M}$

$<0.125$

Level 2-Photon Cuts

L2 EM Cluster $E_{T}$

$>12 \mathrm{GeV}$

L2 EM Cluster $E_{H A D} / E_{E M}$

$<0.125$

L2 EM Cluster $E_{T}^{I S O}$

$<1.0 \mathrm{GeV}$

L2 EM Cluster $|\eta|$

$<1.1$

Level 2-SVT Cuts

L2 SVT number of tracks

$\geq 1$

L2 SVT $\chi^{2}$

$<15$

L2 SVT $P_{T}$

$>2 \mathrm{GeV} / \mathrm{c}$

L2 SVT decay length $D$

$0.120 \mathrm{~mm}<D<1.000 \mathrm{~mm}$

Level 3

L3 EM Cluster $\chi_{C E S}^{2} \quad<20$

L3 Tracking SVT/COT matching tracks $\quad \geq 1$ 
more fully explained in Section 6.2.

Monte Carlo data samples were generated for both ISR and FSR signal events, together with the primary QCD background. Both signal Monte Carlo samples were generated at leading order using MadGraph 4, while the primary QCD background was generated to include next-to-leading order events using MadGraph 5. The nextto-leading order event generation was obtained by explicitly specifying the presence of up to one additional parton in the MadGraph events, and with MLM matching[42] used to eliminate the possibility of double-counting in the Pythia showering. MLM matching is a setting for Monte Carlo generation which allows for the presence or absence of specific processes in the event. Specifically, production of additional elements present in the MadGraph can be removed from the Pythia algorithm to avoid having the same end state being produced in two different ways when the two generators are combined. The most typical means of doing this is to eliminate showering events which include energy above a certain threshold, or elements from a jet which spread to too great of an angle.

The size of a data sample is measured by the integrated luminosity included in the sample, while Monte Carlo data samples are measured by the equivalent integrated luminosity required to obtain that many events with the particular event signature. This analysis is able to use the entire $9136 \pm 548 \mathrm{pb}^{-1}$ of data collected at CDF during Run II. The ISR sample (described as dataset px0s0b in CDF nomenclature) comprises the equivalent of $1682 \mathrm{fb}^{-1}$, roughly 200 times more than the entire CDF Run II dataset. The FSR sample (dataset px0s1b) is the equivalent of 3045 $\mathrm{fb}^{-1}$, and the QCD $\gamma b \bar{b}$ sample is equivalent to $45.3 \mathrm{fb}^{-1}$. As the QCD processes that generate that particular sample are far more frequent than the $Z \gamma$ production processes, the equivalent integrated luminosity is far smaller for a comparable number of events. As ISR is more kinematically distinct from the irreducible QCD 
background, this analysis is optimized to preferentially select ISR.

\subsection{Trigger efficiency}

One of the realities of collider physics is that every time a trigger is used to select interesting events, some events which should be included fail to activate for assorted reasons. Therefore, this analysis models the trigger behavior by considering a related data sample. The portion of the trigger which is dependent upon the $\gamma$ kinematics can be modeled by imposing its restrictions on the Monte Carlo data, and by increasing the $E_{T}^{\gamma}$ cut from 12 to $15 \mathrm{GeV}$, so this requirement is imposed upon the Monte Carlo, and need not be simulated. One important note is that the cut on $E_{T}^{i s o}$ was changed during run period 0 from $3 \mathrm{GeV}$ to $1 \mathrm{GeV}$, so this analysis applies the latter cut to both data and Monte Carlo to enforce consistency in this trigger.

In order to evaluate the efficiency of the SVT trigger, this analysis considers the inclusive photon dataset $c p h 1$, which includes effectively all central isolated photons with $E_{T}^{\gamma}>25 \mathrm{GeV}$. Selecting events with at least one $b$-tagged jet $\left(E_{T}^{j}>15\right.$ $\left.\mathrm{GeV},\left|\eta^{j}\right|<1.5\right)$, the number of events which pass the SVT trigger is compared to the total number of events present in the inclusive photon dataset. As no $b$ tagging algorithm is completely pure or efficient (see Section 6.4 for a more thorough discussion of $b$-tagging), this analysis uses profile matching on the secondary vertex mass to estimate the contribution of light flavor $(u, d, s)$ jets, charm jets, and $b$ jets to the total secondary vertex mass profile for $b$-tagged jets. Profile matching fits the distribution of a parameter in data to a combination of the distributions for Monte Carlo simulations of each element present in the data.

To determine the efficiency of the trigger, the number of $b$ flavor jets tagged as such in the inclusive photon dataset (as shown by the fit in Figure 6.2) is compared to the estimated number of $b$ flavor jets present in the entire dataset, without 


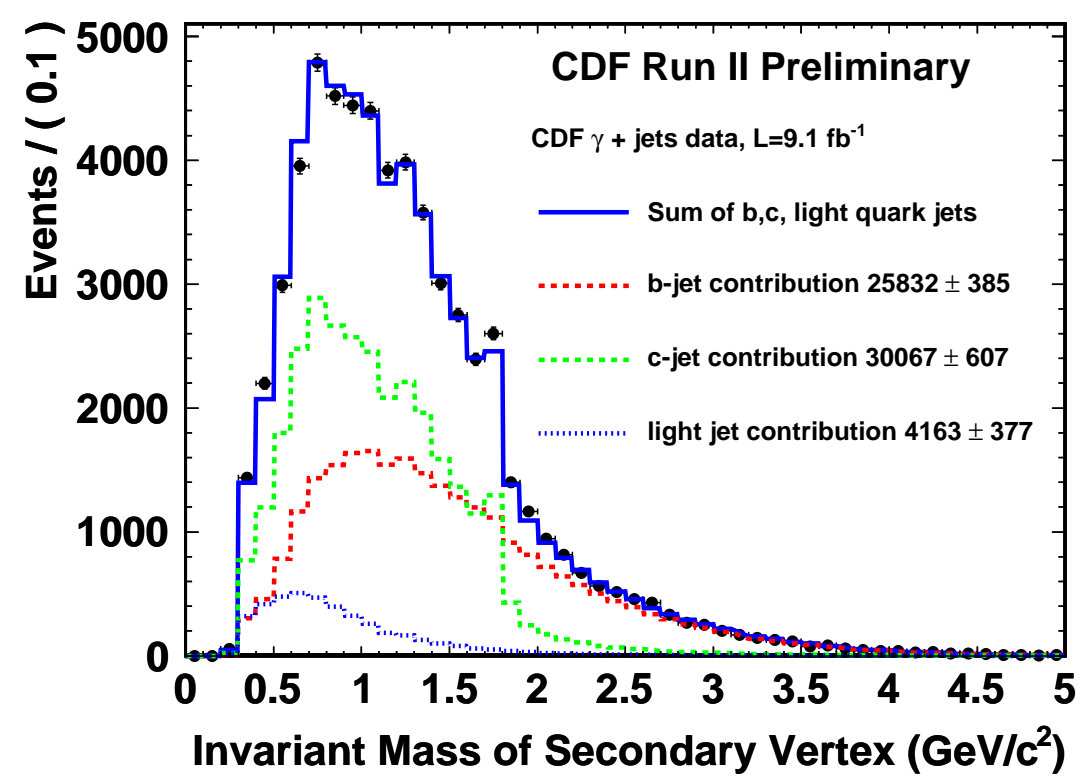

Figure 6.2: Mass associated with secondary vertex for jets passing inclusive $\gamma$ trigger and SVT trigger, jet $E_{T}$ between 20 and $140 \mathrm{GeV}$.

requiring the SVT trigger. As both of these quantities are determined by fitting templates, for the highest energy events, more events were reconstructed as having $b$ quarks in the data with the SVT trigger than in the inclusive data. The large statistical and systematic errors associated with this energy range help to explain this obviously unphysical result.

The secondary vertex mass templates were obtained from Monte Carlo simulation (generated using Pythia). The trigger efficiency was calculated as a function of $E_{T}^{j}$, and is shown in Figure 6.3, the efficiency shows a slight increase at higher $E_{T}^{j}$, but is consistent with being constant over the entire region. In order to estimate the systematic uncertainty is this calculation, the Monte Carlo secondary vertex mass templates were shifted by $\pm 3 \%$, in accordance with estimated systematic uncertainty in the jet energy scale at CDF[43]. All further Monte Carlo samples will be 
weighted according to this fit for trigger efficiency in order to simulate the efficiency of the SVT trigger, based on the $E_{T}$ of their leading jet.

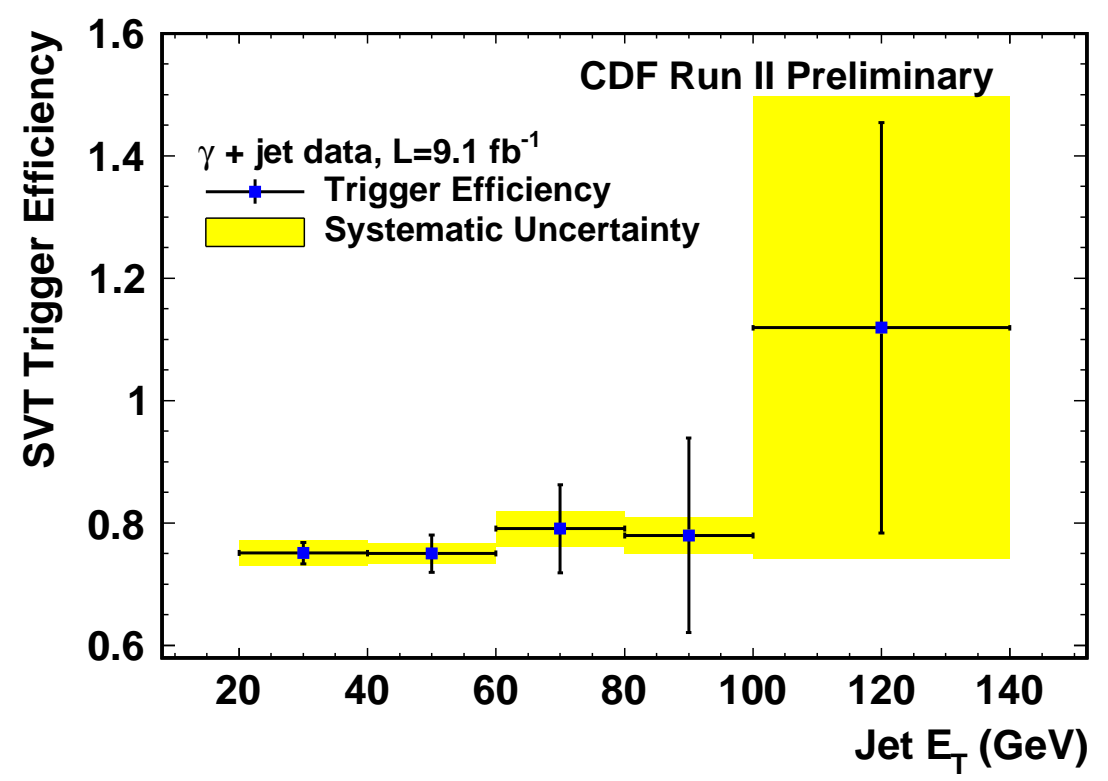

Figure 6.3: SVT trigger efficiency as a function of jet $E_{T}$ in stored data.

\subsection{Photon identification}

Since photons are uncharged particles, they leave no detector tracks, nor are they deflected by magnetic fields. Thus, in the CDF detector, photons are not observable in any tracking region, and are instead identified by deposits of energy in the electromagnetic calorimeter, together with an absence of a track associated with the energy deposit. Furthermore, as shown in the trigger requirements (given in Table 6.1), a significant amount of energy present in the hadronic calorimeter in the vicinity of the photon candidate is used as an additional veto.

Because this signature can be produced by the decay of certain hadrons (such as $\pi^{0} \rightarrow \gamma \gamma$ ), CDF has developed as standard photon identification artificial neural 
network[36], which attempts to distinguish between prompt photons (those associated with the primary vertex) and photons which are the product of a hadronic decay (noting that it is possible for multiple photons to be incorrectly reconstructed as a single event if they are sufficiently close to each other). This neural network considers isolation energy (energy in a region near the photon candidate, the ratio of energy deposited in the hadronic and electromagnetic calorimeters by the candidate particle, and the momentum of all particles in a region near the photon candidate, among other experimentally determined parameters. This analysis requires that the photon ID ANN output be > 0.85, which corresponds to roughly $90 \%$ signal efficiency and $85 \%$ background rejection[36]. (See Table 6.2 for the list of full cuts).

\section{$6.4 \quad b$-Jet identification}

As noted in Chapter 2, lone quarks generate a shower of particles known as a jet. Since $b$ jets are generated by the production of a high $p_{T} b$ quark, they must include at least one hadron with a $b$ quark. As the $b$ quark is substantially more massive than the first two generations of quarks, any hadron containing one must also be ceteris parabis more massive than hadrons typically associated with lighter flavor quarks. Since, in general, more massive particles decay more rapidly than less massive ones, most $b$-tagging algorithms search for the presence of a secondary decay vertex near the primary interaction point.

This analysis uses the Tight SecVtx b-tagger[44][27], which is one of the standard algorithms used to isolate $b$ jets in CDF analyses. Compared to the other standard, general-use algorithms, Tight SecVtx reduces efficiency in favor of greater purity. Given the relative number of light flavor jets that pass the SVT trigger relative to the number of $b$ flavor jets, this is a desirable property. It is worth noting that the Higgs group at CDF found it necessary to construct their own b-tagging algorithm (HOBIT) to better suit their search for $H \rightarrow b \bar{b}$ events[45]. Noteworthy 
is that HOBIT is itself a neural network, and the output of the HOBIT algorithm can be used as an analysis cut, based on the desired purity and acceptance rates. For the same purity as Tight SecVtx, HOBIT has roughly $40 \%$ higher acceptance. Unfortunately, the HOBIT algorithm was not used in this analysis, as its use had not generally spread beyond the Higgs group.

\subsection{Kinematic cuts}

To further reduce the number of QCD events present in the data sample, this analysis imposed further kinematic cuts which had minimal impact on the Monte Carlo sample of ISR events. The full list is shown in Table 6.2, but at least a few of these merit specific explanations. Since the trigger requires $E_{T}^{\gamma}>12 \mathrm{GeV}$, this cut is increased to $15 \mathrm{GeV}$ for the analysis, to reduce the impact of trigger effects on the data sample. As ISR $\gamma Z$ events typically have higher energy than QCD $\gamma b \bar{b}$ events, tighter analysis cuts are imposed here. Furthermore, the invariant dijet and three-body masses have cuts imposed to isolate the region closer to the $Z$ mass. Specifically, only events with a dijet mass between 50 and $110 \mathrm{GeV}$ are kept, and a minimum three-body mass of $80 \mathrm{GeV}$ is required. As the nominal $Z$ mass is 91 $\mathrm{GeV}$, and as the distribution of $E_{T}^{\gamma}$ falls off rapidly for all samples involved, these cuts should preserve nearly all signal events, while reducing background events. The cuts on $|\eta|$ are imposed to restrict the data sample to the central region of the detector, where such events are most precisely measured. The angular separation $\left(\Delta R=\left((\Delta \eta)^{2}+(\Delta \phi)^{2}\right)^{0.5}\right)$ cuts are imposed to reduce that possibility of inaccurate reconstructions, more so than to reduce the presence of QCD $\gamma b \bar{b}$ events. 
Table 6.2: Summary of cuts implemented in this analysis, excluding trigger requirements.

\begin{tabular}{lc}
\hline$E_{T}^{\gamma}$ & $>15 \mathrm{GeV}$ \\
Leading jet $E_{T}$ & $>30 \mathrm{GeV}$ \\
Secondary jet $E_{T}$ & $>20 \mathrm{GeV}$ \\
$m_{j j}$ & $50<m_{j j}<110 \mathrm{GeV}$ \\
$m_{\gamma j j}$ & $>80 \mathrm{GeV}$ \\
$\Delta R\left(\gamma, j_{1}\right)$ & $>0.7$ \\
$\Delta R\left(\gamma, j_{2}\right)$ & $>0.7$ \\
$\Delta R\left(j_{1}, j_{2}\right)$ & $>1.5$ \\
Jet $|\eta|$ & $<1.5$ \\
$\left|\eta^{\gamma}\right|$ & $<1.0$ \\
\hline
\end{tabular}




\section{CHAPTER 7 \\ DATA MODELING}

For events that pass our trigger and analysis cuts, with a $\gamma$ candidate and two b-tagged jets, there are still four classes events which produce this signature. In addition to the signal $Z \gamma$ events, there are also QCD $\gamma b \bar{b}$ events, as well as events where at least one element is misidentified. These fall into two main categories: events where the $\gamma$ candidate is not actually a photon associated with the primary vertex, and events where at least one of the $b$-tagged jets does not actually contain a $b$ quark. An overview of each of these categories, and how they are modeled, follows.

\subsection{Monte Carlo datasets}

In order to distinguish $\gamma b \bar{b}$ events that arise from $Z$ decays from those generated by QCD processes, this analysis produced Monte Carlo data samples corresponding to each particular process. For Initial State Radiation $(p \bar{p} \rightarrow Z \gamma \rightarrow \gamma b \bar{b})$, a Monte Carlo sample equivalent to $1684 \mathrm{fb}^{-1}$ was generated using MadGraph version 4[39]. In CDF nomenclature, this dataset is described as px0s0b. The Monte Carlo sample for Final State Radiation $(p \bar{p} \rightarrow Z \rightarrow \gamma b \bar{b})$ was generated in the same manner, but due to the lower cross section for this process, the sample was equivalent to $3045 \mathrm{fb}^{-1}$, falling into the category known as px0s1b at CDF.

The irreducible QCD background, consisting of all events $p \bar{p} \rightarrow \gamma b \bar{b}$, with $Z$ production explicitly forbidden, was simulated by a Monte Carlo sample generated using MadGraph version 5, where up to one additional parton was explicitly permitted in the MadGraph events, and MLM matching was used to eliminate the possibility of double-counting in the Pythia scattering (that is, additional events with the equivalent of exactly one additional parton were not generated in the Pythia showering process)[42]. The final Pythia showering process is fed into a simulation 
of the CDF detector, which uses GEANT version 4[41] software to imitate the observation of the particles present in the shower as they would appear in the CDF detector. As this process is far more common the $Z \gamma$ production, despite the larger dataset size, it is still equivalent to only $45.3 \mathrm{fb}^{-1}$ of data. CDF designates this data sample as $p x 0 s 9 b$ for internal notation. The more generalized case of production of photons in association with one or more $b$ jets is an established parameter for CDF $\operatorname{data}[46]$.

\subsection{Misidentification of photon candidates}

In order to estimate the number of events where the $\gamma$ candidate is not a prompt photon, it is necessary to model the number of non-prompt photons that pass the photon ID neural network cut. Since the QCD Monte Carlo data sample includes both prompt and non-prompt photons, it was used to generate templates of photon ID neural network output for these two categories of events. These two templates are then used to fit the data profile for photon ID neural network output, yielding an estimate of the number of decay product $\gamma$ pass the photon ID cut. This plot is shown in Figure 7.1, and is used to estimate that $135 \pm 31$ fake $\gamma$ events are present in the $\gamma+$ jets data and will pass the photon ID ANN cut.

To evaluate the systematic uncertainty in this estimate, two independent methods were used. The first method was to shift photon ID ANN output by \pm 0.025 (which is one half the size of the bins used in the fit), then constraining it to the interval between 0 and 1 (all events which would thereby leave this range were set to the given endpoint). The second method was to fit a rebinned version of the distribution consisting of only three bins: a bin that passed the cut $\gamma$ ID ANN $>0.85$; a bin of events clearly not prompt $\gamma(\mathrm{ANN}$ output $<0.15)$; and a bin of intermediate events $(0.15<\mathrm{ANN}$ output $>0.85)$. Combining these two uncertainties in quadrature to obtain the total systematic uncertainty, this analysis calculates 
that, out of 1555 data events, $134.7 \pm 9.0$ (stat.) \pm 29.8 (sys.) are due to non-prompt photons being reconstructed as prompt photons and passing the photon ID ANN cut. The rebinning sorts photon candidates into those that pass the analysis cut, those that constitute the sideband for this background, and those that are least likely to be actual photons.

In order to model the effect of the template shape of this particular background on the remainder of the analysis, a data sideband was used. This sideband is fixed to the calculated normalization, and comprises data events which pass all other analysis cuts, but which have a photon ID neural network output between 0.15 and 0.85. This will be referred to as the fake $\gamma$ sideband.

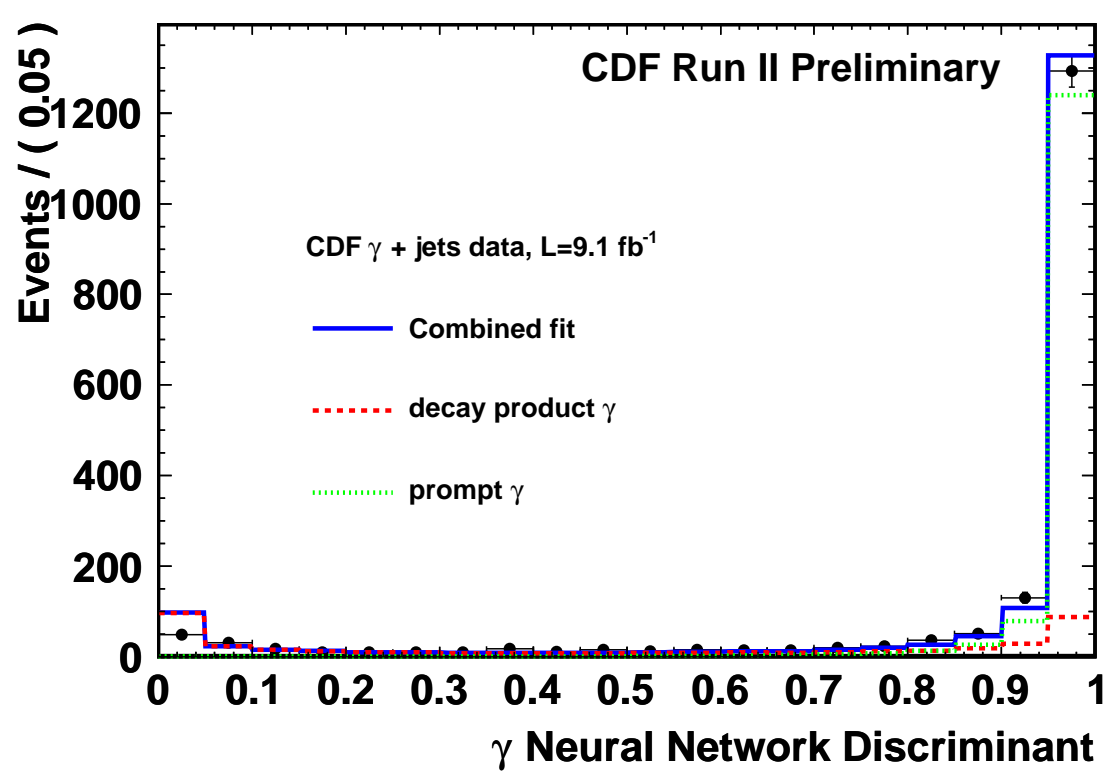

Figure 7.1: Fitted $\gamma$ identification neural network output. 


\subsection{Incorrectly tagged jets}

The Tight SecVtx tagger, despite its name, is not completely efficient at eliminating all jets which lack a $b$ quark[27]. In order to correct for this, the twodimensional plot of secondary vertex mass for the two leading jets (secondary vertex mass for the second jet versus secondary vertex mass for the leading jet) is obtained for the data sample, the QCD Monte Carlo, and the fake $\gamma$ sideband. The data distribution is then fitted to these templates, with the normalization of the fake $\gamma$ sideband fixed (as given in Section 7.2), and with the light jet content determined by the fitting procedure. Systematic uncertainty was obtained by varying the secondary mass vertex by $\pm 3 \%$ for the Monte Carlo templates (as used for the rest of this analysis), and observing how the estimated number of incorrectly tagged light jets changed. Based on this procedure, an estimated $358.6 \pm 52.1$ (stat.) \pm 124.5 (sys.) events with light flavor jets incorrectly tagged as $b$ jets are present in the data for this analysis. For the final neural network fit, the presence of these incorrectly tagged jets is modeled using a data sideband which passes all other analysis cuts, but which has exactly $0 b$ tagged jets. 


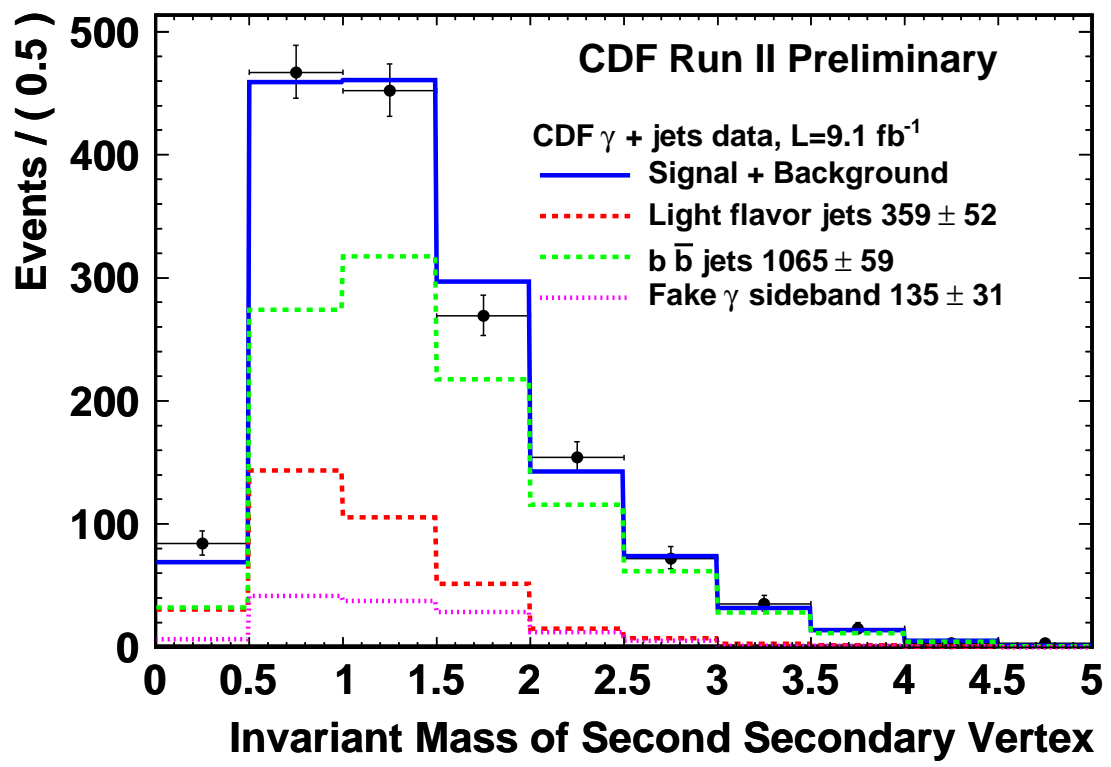

Figure 7.2: Fake $b$ normalization estimated using secondary vertex mass. Onedimensional projection of secondary vertex mass distribution (in $\mathrm{GeV}$ ) fit for leading jet $E_{T}$ between 20 and $140 \mathrm{GeV}$. 


\section{CHAPTER 8 SIGNAL YIELD CALCULATION}

As shown in Figure 8.1, the $m_{j j}$ distribution has insufficient separation between signal and background to obtain a meaningful result by fitting mass templates. Therefore, this analysis utilizes a neural network to distinguish between ISR and the primary QCD $\gamma b \bar{b}$ background. This neural network is applied to the data sample, and the output of the neural network is fitted to templates of neural network output based on Monte Carlo samples and the relevant sidebands. While the final results show that significant statistical uncertainty remains, this helped to reduce the systematic uncertainty associated with the jet-energy scale. Additionally, the preliminary mass profile fits showed extreme fluctuation in estimated signal yield and fraction (as well as signal ISR/FSR composition) as energy scale cuts were adjusted, casting significant doubt on any attempt to use the mass profile directly in the yield estimation. In order to convert an estimated signal yield into a cross section, we determine the rate at which events pass through each stage of this analysis, including $b$-tagging algorithms and kinematic cuts.

8.1 Neural network development

To determine the most effective method of constructing a neural network to distinguish between signal and background, several factors were considered. Construction of this neural network was a major part of this analysis. First of all, the syntax for developing an artificial neural network is well-developed in the ROOT code libraries. Using predefined functions, a type of artificial neural network known as a multilayer perceptron can be developed, with input variables, network structure, and number of training iterations left for the user to define. In addition to training a neural network to distinguish between QCD $\gamma b \bar{b}$ production and ISR $\gamma Z$ production, these functions show the relative weights of different variables, along 


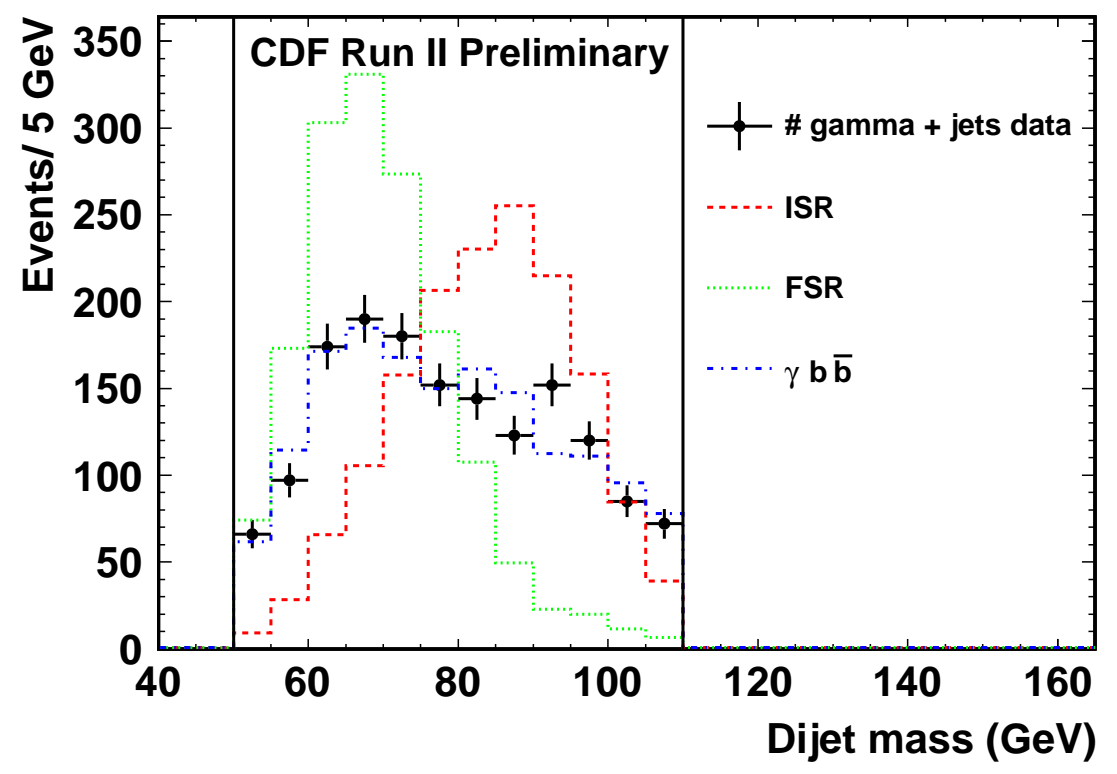

Figure 8.1: Dijet mass distribution for data and various Monte Carlo samples (Monte Carlo templates normalized to data).

with connections between various nodes, and show the output template for the training sample. Following standard convention, the background QCD $\gamma b \bar{b}$ sample was set to have neural network output values closer to 0 , with signal $\gamma Z$ production having values closer to 1 .

Perhaps the most important step in developing a neural network is to properly select input observables. For every input parameter, the data sample should be reasonably well modeled by the Monte Carlo samples (as QCD $\gamma b \bar{b}$ production is far more common than $\gamma Z$ production, the QCD sample should more closely reflect the data), and there should be some reasonable distinction between the two Monte Carlo samples[35].

In a previous search for hadronic decays resulting from $\gamma Z$ production at CDF [33], variables had to be chosen which would not artificially distinguish between 
different flavors of final state jets, as the search included all hadronic final states. Thus, this previous analysis chose variables that were independent of the mass scale, and required more detailed modeling.

Because the present analysis did not construct next-to-leading order samples for both signal and irreducible background, certain parameters used in the previous analysis were not applicable here, as they would be artificially biased by the absence of next-to-leading order events in one of the samples. However, by restricting final states to heavy-flavor decays, this analysis is able to make direct use of mass and energy information as input variables. The following 8 variables were selected as inputs:

- $m_{j j}$, reconstructed dijet mass

- $m_{\gamma j j}$, reconstructed three-body mass

- $E_{T}^{j 1}$, leading jet transverse energy

- $E_{T}^{j 2}$, secondary jet transverse energy

- $\Delta R\left(\gamma, j_{1}\right)$, separation between leading jet and photon

- $\Delta R\left(\gamma, j_{2}\right)$, separation between secondary jet and photon

- $\Delta \phi_{j j}$, difference in azimuthal angle between jets

- $\Delta \eta_{j j}$, difference in pseudorapidity angle between jets

Determining the most effective neural network architecture is not a precise procedure. As mentioned in Section 5.1, the value of a particular neural network is based on its utility in providing a reliable and accurate fit. After a certain amount of trial and error, the multilayer perceptron architecture was finalized with two layers of hidden nodes (the first containing twelve nodes, and the second six). To identify 
possible overtraining, only half of the available Monte Carlo events were used to train the network, with the other half kept in reserve to provide a check on the ability of the network to separate other events in the same distributions. The input variables, coupled with their relative weights, along with network architecture and template shape, are found in Figure 8.2. The top plot details the neural network architecture, the middle plot the relative weights of the input variables, and the bottom plot shows the template shapes for the signal and background Monte Carlo samples.

\subsection{Neural network fitting}

In order to obtain an estimate of signal yield, the neural network output, as applied to the data sample, is fitted to four templates, each reflecting a portion of the events which produce the $\gamma b \bar{b}$ signature. The normalization of the fake $b$ and fake $\gamma$ sidebands was fixed by their estimated presence, as described in Chapter 7. The signal population is modeled using a template consisting of $90 \%$ ISR and $10 \%$ FSR, based on the relative MadGraph cross section for the analysis cuts. The data output is then fitted with the normalization of the Monte Carlo signal and QCD background templates determined by the RooFit analysis package, as shown in Figure 8.3.

From this plot, it is apparent that there is significantly more QCD $\gamma b \bar{b}$ background than $\gamma Z$ signal. The fit results are summarized in Table 8.1. The sidebands are normalized based on predicted presence, while $\gamma b \bar{b}$ and $\gamma Z$ content is determined by the fitting algorithm. It is important to note that (while not listed explicitly here) the statistical uncertainty in the signal presence in this fit is still quite large relative to the signal yield.

Statistical uncertainty inherent in the neural network fit dominates the analysis, and suggests that a more finely tuned neural network might be able to improve 


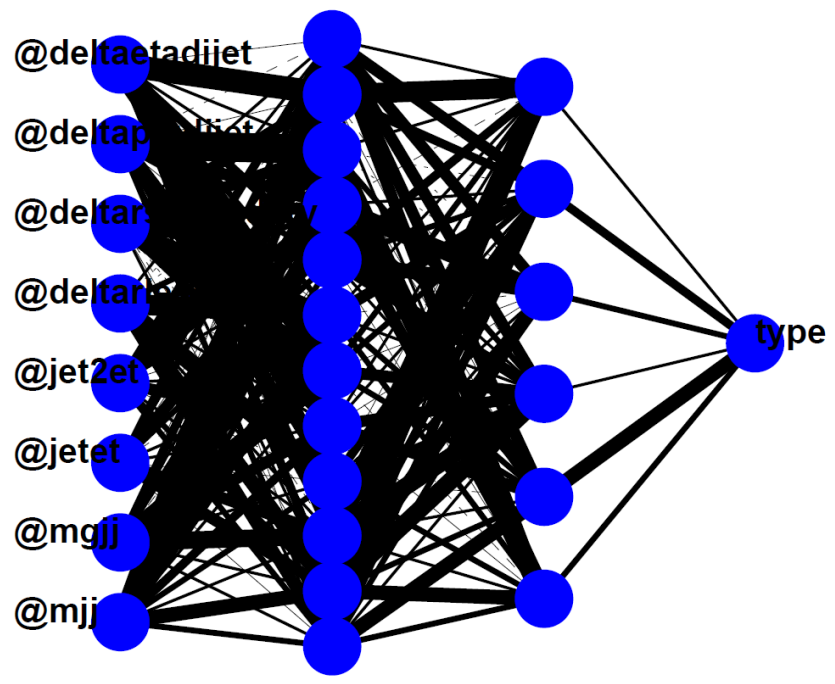

(a) Neural network architecture

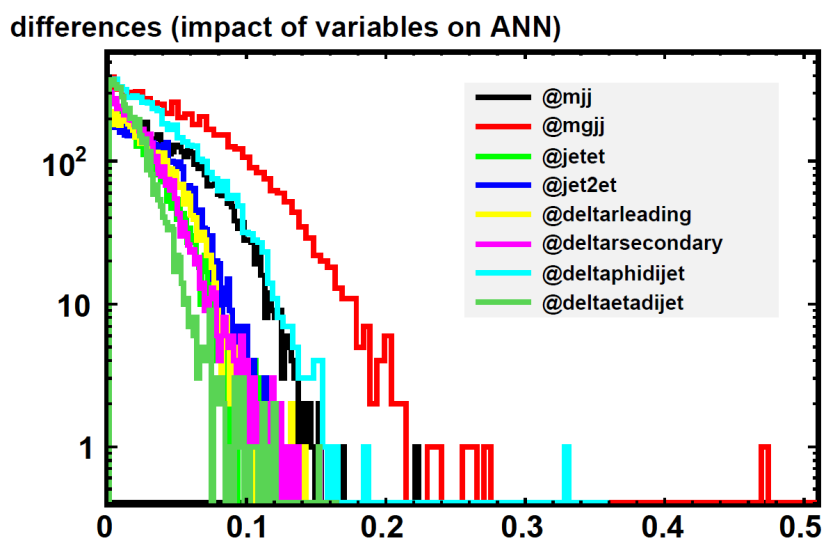

(b) Neural network input variable weight

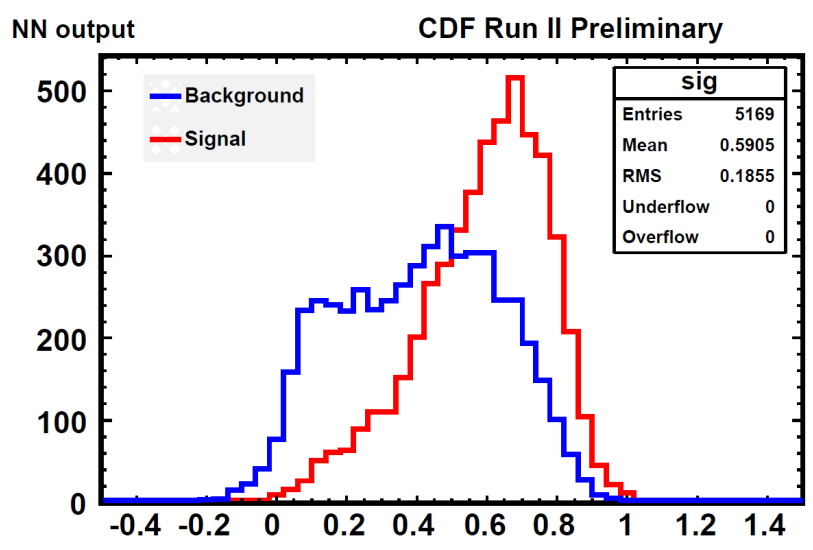

(c) Neural network signal and background template shape

Figure 8.2: Plots detailing neural network information. 


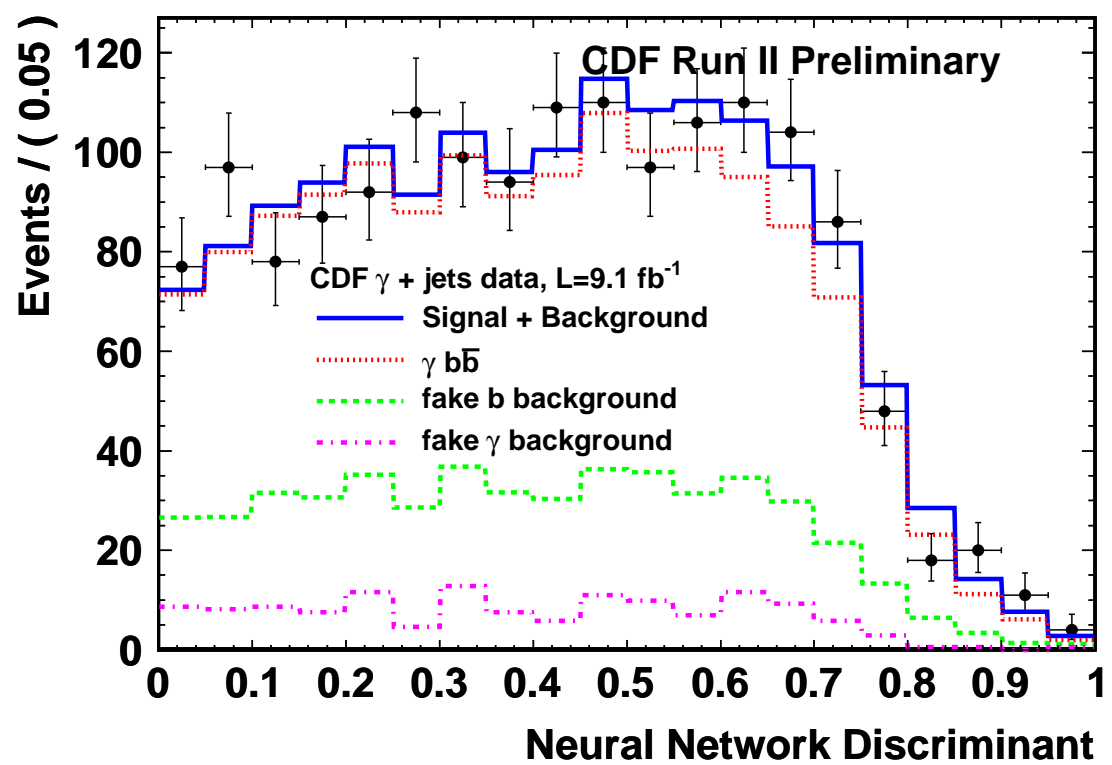

Figure 8.3: Fit of neural network output, with components shown stacked on the plot.

upon this analysis. At no point during the process of developing the neural network was such an improvement definitively identified, so it might be necessary to have next-to-leading order events in all Monte Carlo samples, in order to incorporate more analysis variables. The quality of the fit is illustrated in Figures 8.4 through 8.9, where the various data elements are normalized in accordance with their presence in this fit, as shown in Table 8.1. Qualitatively, these kinematic variables appear to be in reasonable agreement between the fit and the data.

\subsection{Unfolding factor}

In order to convert signal yield into a cross section measurement, it is essential to determine the frequency at which signal events are recorded in the detector, and thereby incorporated into the data sample. Cross section is related to signal yield by the equation $\sigma=N / L \epsilon$, where $\sigma$ is the cross section, $N$ the number of events 
Table 8.1: Elements of (uncorrected) neural network fit.

\begin{tabular}{cr}
\hline Fit Component & Number of Events \\
\hline Fake $\gamma$ sideband & $134.7 \pm 9.0$ (stat. $) \pm 29.8($ sys. $)$ \\
Fake $b$-jet sideband & $358.7 \pm 52.1$ (stat. $) \pm 124.5($ sys. $)$ \\
Fitted $\gamma b \bar{b}$ & $956.1 \pm 55.0($ stat. $)$ \\
Fitted $\gamma Z$ & $106.4 \pm 53.2($ stat. $)$ \\
\hline
\end{tabular}

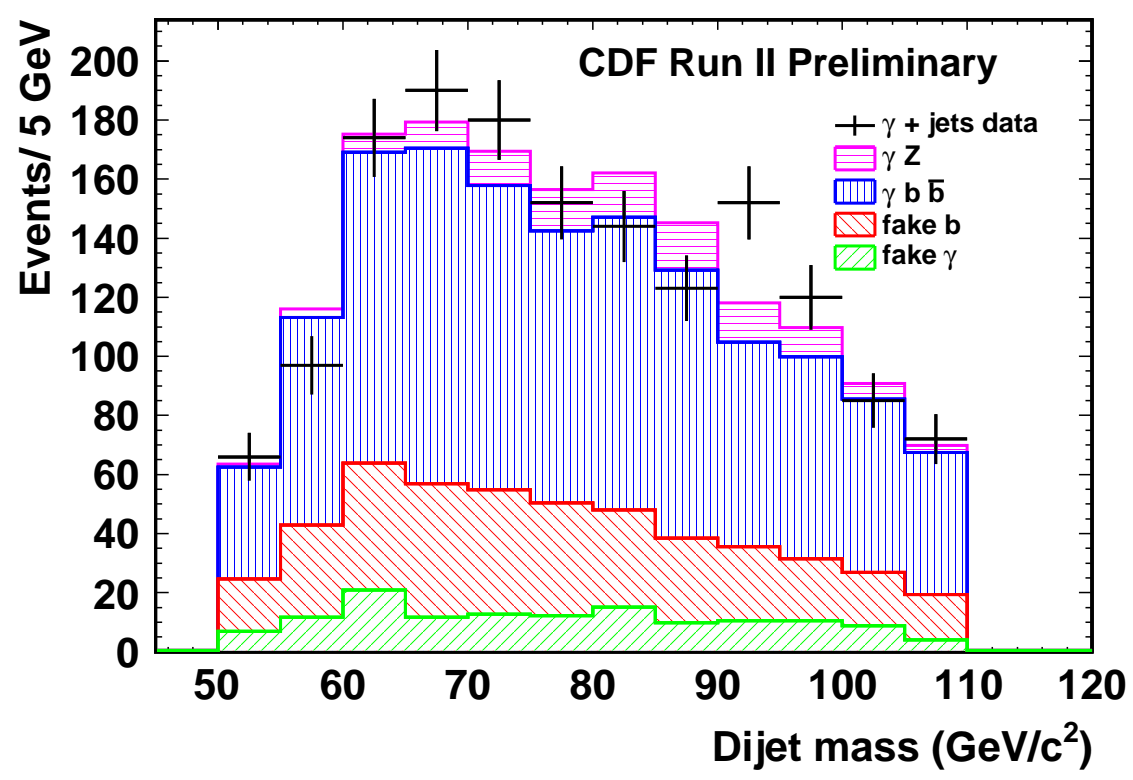

Figure 8.4: Reconstructed $m_{j j}$ for composite fit. 


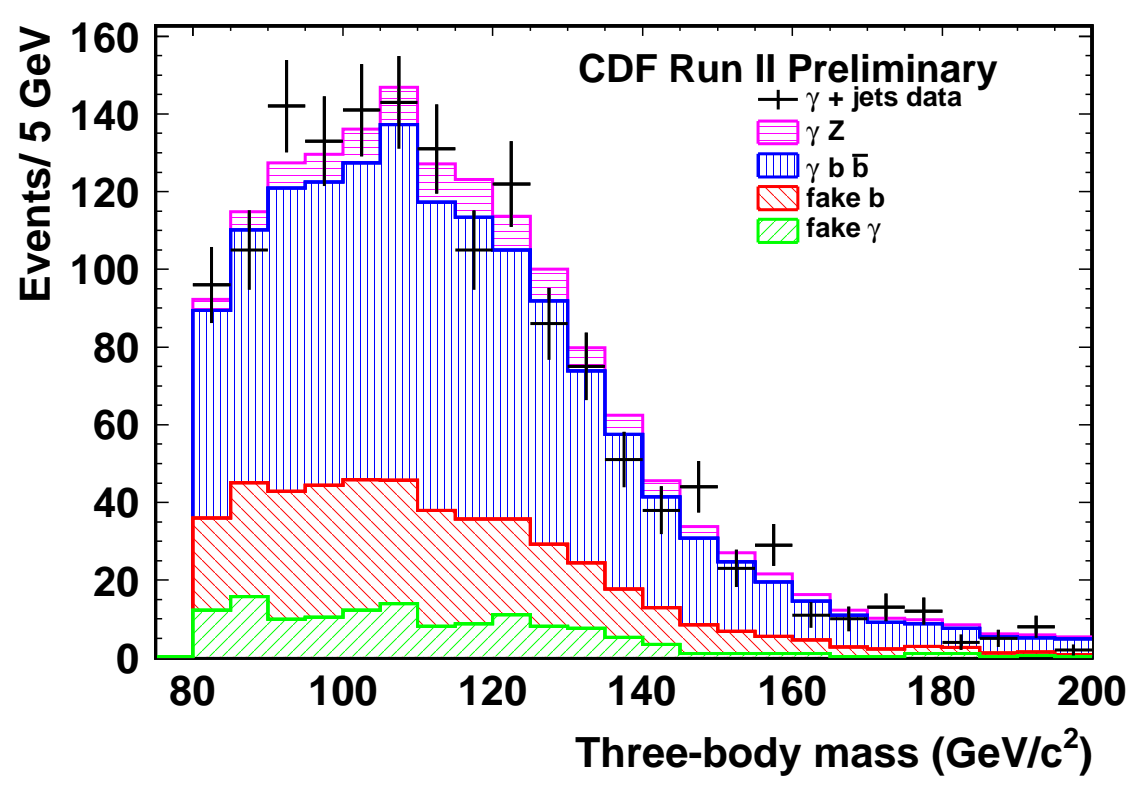

Figure 8.5: Reconstructed three-body invariant mass for composite fit.

in the signal yield, $L$ the integrated luminosity, and $\epsilon$ the unfolding factor (i.e. the acceptance rate for signal events).

Since the unfolding factor, $\epsilon$, incorporates all of the trigger and selection efficiencies, it is readily calculated by determining the number of signal Monte Carlo events which pass all of the kinematic cuts at the parton level, and comparing that to the number of reconstructed events that satisfy the same cuts, based on the same original sample. For the sake of obtaining a better understanding of the unfolding factor, the unfolding factor is shown in Figure 8.10 as a function of $E_{T}^{\gamma}$.

This plot was obtained by dividing two histograms of $E_{T}^{\gamma}$, each of which was derived from the same Monte Carlo sample for signal events. This sample incorporates both ISR and FSR, weighted according to their relative cross sections. The numerator is simply those events in the Monte Carlo sample that pass the analysis cuts, as weighted for SVT trigger efficiency. The denominator is those Monte Carlo events 


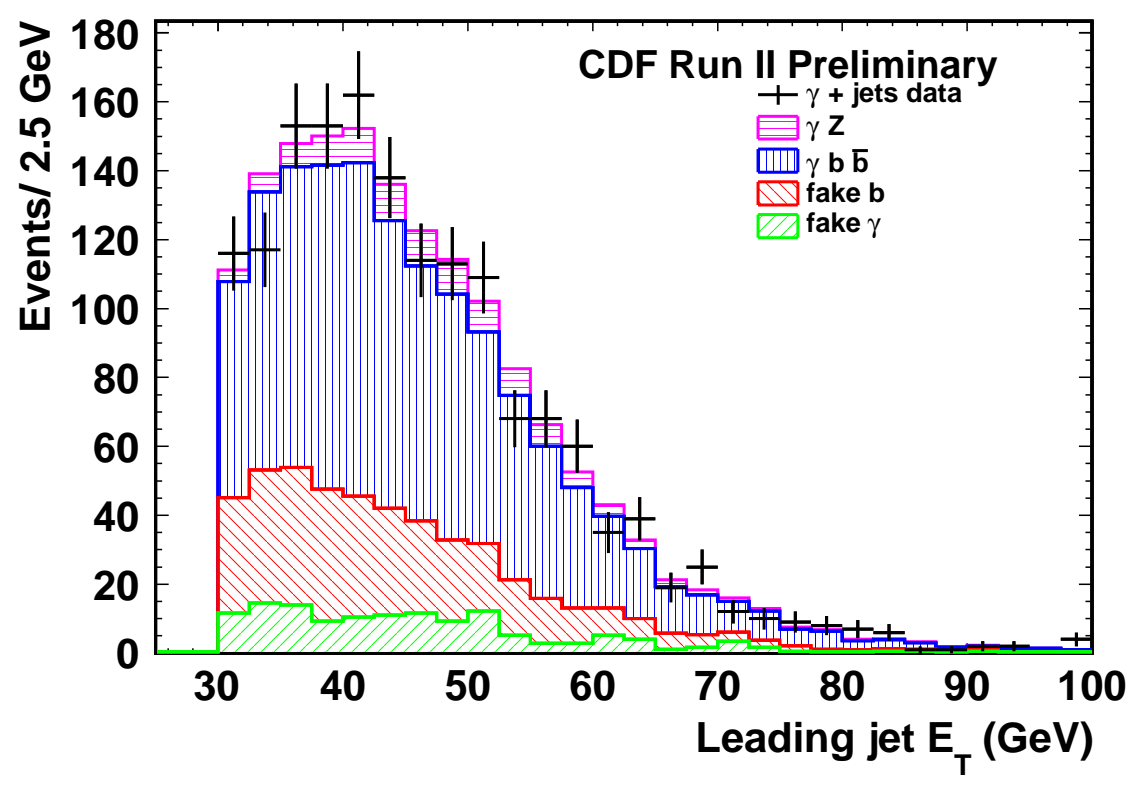

(a) Leading jet $E_{T}$

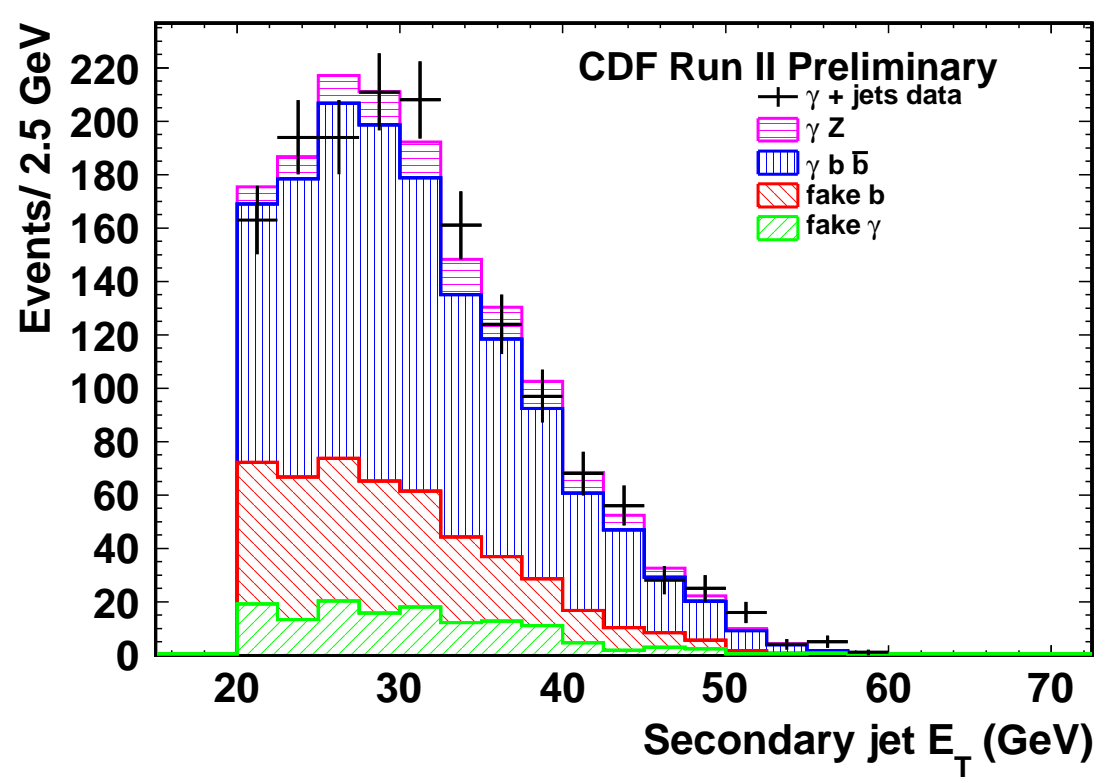

(b) Secondary jet $E_{T}$

Figure 8.6: Transverse energy associated with each jet, normalized to match composite fit. 


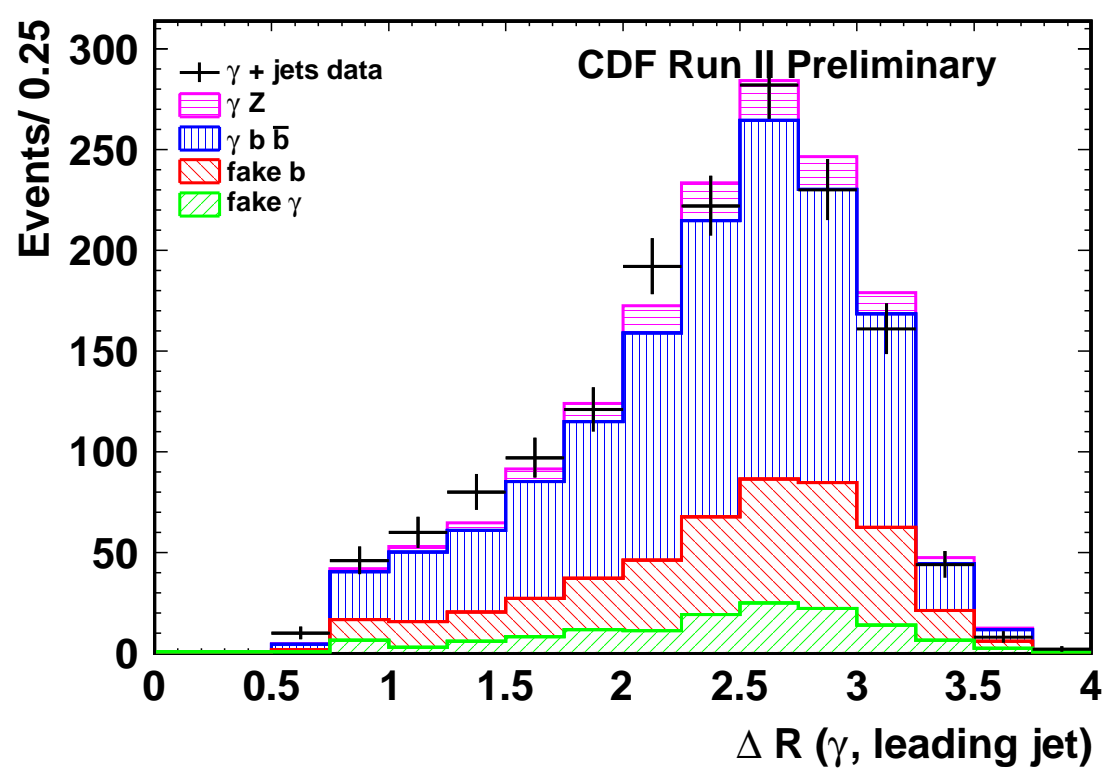

(a) $\Delta R(\gamma$, leadingjet $)$

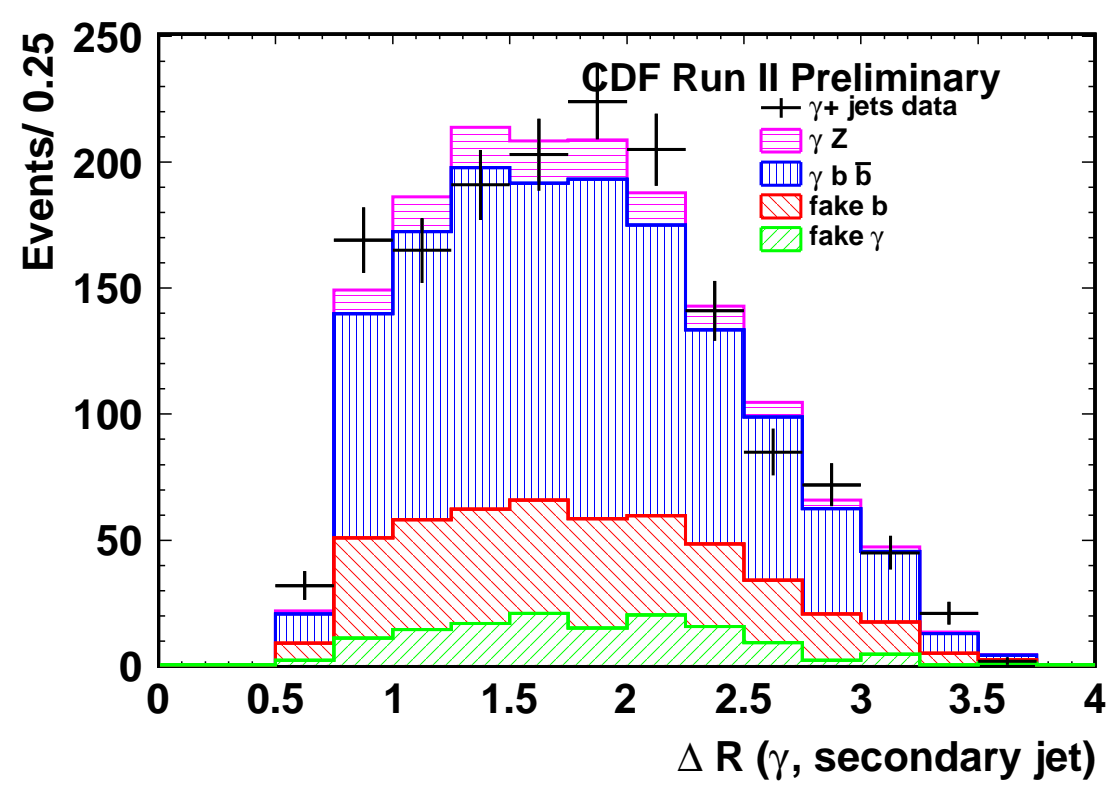

(b) $\Delta R(\gamma$, secondaryjet $)$

Figure 8.7: Angular separation between $\gamma$ and jets, normalized to match composite fit. 


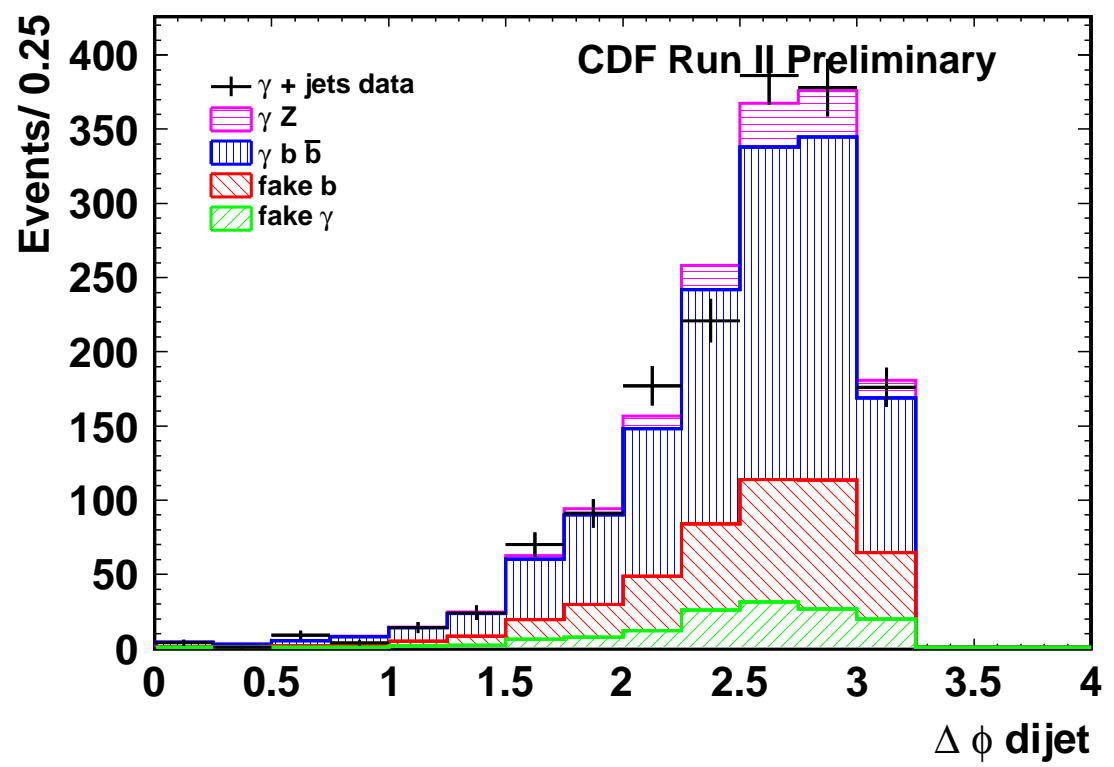

Figure 8.8: $\Delta \phi$ between the two jets using composite fit normalization.

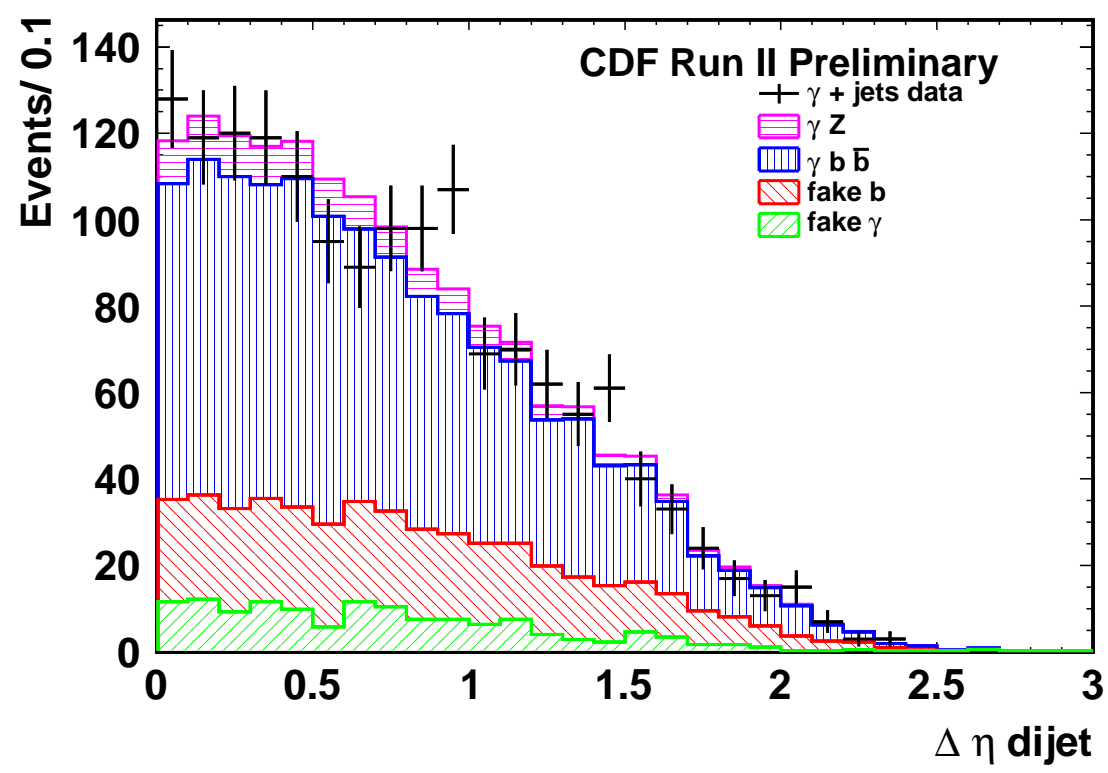

Figure 8.9: $\Delta \eta$ between the two jets using composite fit normalization. 
where the generator level information satisfied the kinematic cuts $\left(\gamma E_{T}>15 \mathrm{GeV}\right.$, $E_{T}^{i s o}<1 \mathrm{GeV},\left|\eta^{\gamma}\right|<1$, jet $|\eta|<1.5$, leading jet $E_{T}>30 \mathrm{GeV}$, secondary jet $E_{T}>20 \mathrm{GeV}, 50 \mathrm{GeV}<m_{j j}<110 \mathrm{GeV}, m_{\gamma j j}>80 \mathrm{GeV}, \Delta R_{j \gamma}>0.7$, and $\left.\Delta R_{j j}>1.5\right)$

Figure 8.10 shows the likelihood that a given event with the appropriate kinematic properties at the parton level will pass the full analysis cuts when reconstructed, plotted as a function of $E_{T}^{\gamma}$. This unfolding factor is calculated, when integrated over the entire sample, to be $\epsilon=0.0362 \pm 0.0003$ (stat.) \pm 0.0049 (sys.). Statistical uncertainty is based on standard counting statistic uncertainties, while systematic uncertainty is detailed in Section 9.4. Note that the unfolding factor decreases as a function $E_{T}^{\gamma}$, a feature which is likely a result of higher energy photons penetrating deeper into the calorimetry and being more likely to fail to pass the trigger or photon ID ANN cuts.

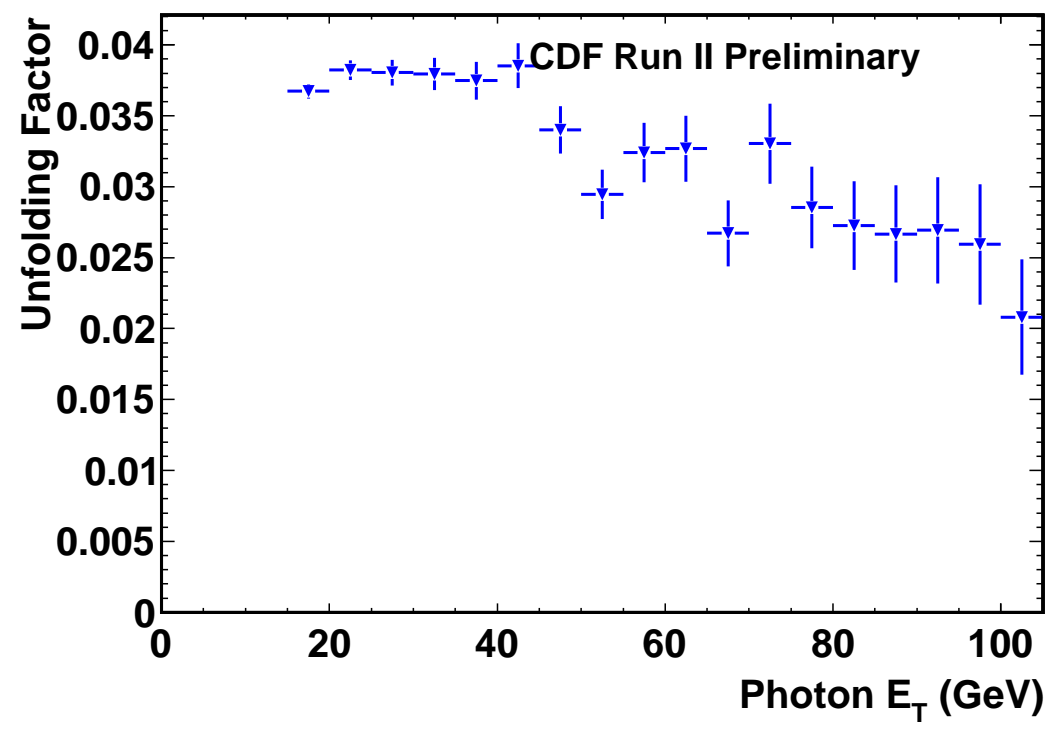

Figure 8.10: Unfolding factor versus Photon $E_{T}$. 


\section{CHAPTER 9 \\ ANALYSIS OF SYSTEMATIC EFFECTS}

In addition to statistical uncertainties, any scientific research must also take into account the possibility of inaccuracies in particular measurements and models used. These effects, which cause any repeated measurement to have similar inaccuracies (as long as the same equipment and models are used), are known as systematic uncertainties. This is in contrast to statistical uncertainties, which are due to fluctuations in data, and which reflect the likelihood of a repeated measurement recording a different result.

For this analysis, systematic uncertainties cover a substantial range of material. Of these, several are deemed sufficiently complex to merit additional discussion. There are, of course, questions as to the most appropriate model used to describe both the photon and light jet sideband, as well as known uncertainties in how well Monte Carlo simulations reflect data. Additionally, some of these uncertainties in Monte Carlo simulation will impact the measurement of the unfolding factor. For convenience, these are listed separately, in order to separate uncertainties in signal yield from uncertainties in total cross section.

Systematic and statistical uncertainties are reviewed and shown numerically in Chapter 10.

\subsection{Fake photon sideband}

The fake photon sideband contributes systematic uncertainty due to uncertainty in both the estimate rate, as well as uncertainties in whether the sideband chosen in the model accurately reflects those events in the analysis sample. To review the results of Section 7.2 , the systematic uncertainty in the rate was estimated in two independent ways, with the total systematic uncertainty derived from the combination of the two methods. 
The first method was to shift the photon identification neural network output and repeat the fit, taking the deviation as a systematic uncertainty based on the accuracy of the photon identification neural network. A more precise method would be to take all of the inputs to the photon identification neural network, and to vary each of those in accordance with its respective uncertainty. Due to the robust ability of the photon identification neural network to distinguish between prompt and nonprompt photons, this was deemed not to be necessary for this analysis. The second method was to rebin the distribution of photon ID ANN output and repeat the fit. Three bins were chosen in order to distinguish those that pass the analysis cut from those that were almost certainly not signal events from those which fell somewhere in between. As previously discussed, this indicated that out of 1555 data events which passed all analysis cuts, $134.7 \pm 9.0$ (stat.) \pm 29.8 (sys.) events contained a $\gamma$ candidate which was not associated with a prompt $\gamma$.

To evaluate the associated uncertainty on the signal yield, the analysis neural network fit was repeated, with the normalization of the fake photon sideband adjusted to the limits of the uncertainty in the fake photon sideband estimate. This was done independently for statistical and systematic errors, in order to distinguish between the two types of error associated with the rate at which $\gamma$ candidates were not due to $\gamma$ production at the primary vertex.

In addition to uncertainty based on the rate of inaccurate $\gamma$ acceptance, there is also uncertainty associated with whether the shape of the sideband accurately reflects those events in the data. The shape uncertainty was calculated by changing the sideband region chosen for the analysis, and thus repeating the fit with a fake photon sideband chosen in a different way. Specifically, instead of using events where the photon identification neural network output was between 0.15 and 0.85 , the sideband was chosen to contain events with photon identification neural network 
output between 0.10 and 0.80 , as a possible alternative band which could have been chosen. When this systematic shape uncertainty was combined with the systematic rate uncertainty for the fake photon sideband, the signal yield changed by about $3 \%$.

\subsection{Light jet sideband}

As mentioned in Section 7.3, the systematic uncertainty in the rate of incorrectly tagged sets was calculated by increasing or decreasing the secondary vertex mass of the Monte Carlo samples by $3 \%$ and repeating the fit[43]. This rate uncertainty was then applied to the normalization of the light jet sideband in the analysis neural network fit, as was done for the fake $\gamma$ sideband. It is also necessary to account for uncertainty in the shape of the light jet sideband.

This particular systematic uncertainty requires more careful attention due to the nature of the Tight SecVtx $b$ tagger. While chosen for its lower fake rate, it has the disadvantage of having a greater number of actual $b$ jets which are not tagged as such. This means that a certain number of $\gamma b \bar{b}$ events will have only one jet $b$ tagged, and some will even have no jets tagged, as shown in Table 9.1.

Normalization for ISR, FSR, and $\gamma b \bar{b}$ is based on MadGraph predictions as well as the actual number of events present in the data with that particular number of $b$ tags. This complicates matters somewhat as the sideband incorporates a certain amount of events that would properly belong to one of our fitted distributions. Thus, in order to evaluate the shape uncertainty, it is only possible to increase the $\gamma b \bar{b}$ presence in the light jet sideband, which is, of course, a change in the opposite direction of an ideal light jet sideband, which would include only light jets. As the $\gamma$ identification cut is far more efficient, this detailed analysis was not required for the fake $\gamma$ sideband.

In order to model the impact of $\gamma b \bar{b}$ presence in this sideband on the signal 
yield, this analysis considered linear combinations of the 0 tag and 1 tag samples (those events which pass all other analysis cuts, but have the stated number of $b$ tagged jets), and determined signal yield as a function of $\gamma b \bar{b}$ presence in the fake $b$ sideband. Not surprisingly, this had an effect on the signal yield, with increasing fractions of $\gamma b \bar{b}$ present in the light jet sideband reducing the fitted signal yield. Rather more surprising was the consistency of the effect, which permitted a linear extrapolation to a hypothetical ideal sideband consisting of only lighter jets. This suggested that if it were possible to obtain such an ideal sideband, that the analysis neural network fit would yield 120.7 signal events. This signal yield was therefore used for calculating final cross section, with its deviation from the actual fit result taken as a systematic uncertainty, associated with the shape of the light jet sideband. Table 9.2 shows this new estimate, as corrected for this observed systematic behavior.

Table 9.1: Expected number of events with a given number of $b$ tags for each Monte Carlo predicted data sample.

\begin{tabular}{ccccc}
\hline Number of $b$ tags & ISR & FSR & $\gamma b \bar{b}$ & data \\
\hline $2+$ & 52.8 & 5.95 & 791 & 1555 \\
1 & 123 & 15.8 & 4196 & 19074 \\
0 & 116 & 19.4 & 7223 & 179058 \\
\hline
\end{tabular}

\subsection{Uncertainties in Monte Carlo reconstruction}

Previous work at the CDF detector has established certain uncertainties in measuring various parameters. These are reflected in differences between the Monte Carlo data sample and the measured dataset for those values. To account for these uncertainties, the values for these observables in the Monte Carlo data sample are 
Table 9.2: Elements of neural network fit, corrected for fake $b$ sideband.

\begin{tabular}{cr}
\hline Fit Component & Number of Events \\
\hline Fake $\gamma$ sideband & 134.7 \\
Fake $b$-jet sideband & 358.7 \\
Fitted $\gamma b \bar{b}$ & 941.8 \\
Fitted $\gamma Z$ & 120.7 \\
\hline
\end{tabular}

shifted according to the uncertainty in detector behavior. While it is possible in principle to shift the values for the data instead, that practice is frowned upon as it performs analysis that does not reflect the values that were measured directly. The convention is to modify the model (the Monte Carlo sample) in accordance with the uncertainty in the measurement, rather than the other way around. These uncertainties impact both estimated signal yield and unfolding factor. This section details the process by which this was calculated, while Section 9.4 details only those effects which only impact the unfolding factor.

We incorporate the uncertainty in the ability of the CDF detector to determine the energy deposited in both the electromagnetic and hadronic calorimeter systems. This imposes uncertainty in the measured photon energy and jet energy, based on the uncertainty in scaling detector hits in the calorimeter to incoming particle energy.

The photon energy scale is relatively well-defined, so we consider the effects of a $1.5 \%$ shift in $E_{T}^{\gamma}$, in accordance with established uncertainties[47]. In this analysis, $\gamma$ energy is only present in the kinematic cuts which determine acceptance. To account for this, the Monte Carlo samples to be used in the fitting process have their photon energy shifted by $1.5 \%$ in order to obtain new templates to be fitted. Additionally, since a change in acceptance rate changes the unfolding factor 
(logically enough, given that the unfolding factor is the total acceptance rate), the unfolding factor is recalculated with the same change in energy.

The change in signal yield is combined with the change in unfolding factor for the same adjustment to photon energy to determine the total impact on the cross section calculation (where the two changes partially cancel each other out). This contributes roughly $1 \%$ total uncertainty to the cross section measurement, which is the smallest systematic effect considered, so further analysis was not deemed necessary.

Jet energy scale is, by constrast, the largest systematic effect for two major reasons: the uncertainty in jet energy scale at CDF is larger than the uncertainty in photon energy; and in addition to impacting kinematic cuts, the jet energy scale enters into the neural network variables used to distinguish signal and background. No less than four neural network variables depend on the jet energy scale, all of which are also used as kinematic cuts $\left(E_{T}^{j e t}\right.$ for both jets, $\left.m_{j j}, m_{j j \gamma}\right)[43]$.

The smaller impact of photon energy scale on $m_{j j \gamma}$ was not included in this analysis, as not only is the uncertainty smaller, but the photon energy has a smaller contribution to the total invariant mass. In order to more precisely determine the impact of the jet energy scale, two different methods of evaluating the change in signal yield were compared. The first method simply repeated the fit with the input parameters for the Monte Carlo events shifted in accordance with the scale uncertainties (event reconstruction at CDF, both for data and Monte Carlo samples, incorporates jet energy uncertainty as a parameter).

The second method is to fit the shifted Monte Carlo templates to the unbiased fit result profile for the same templates (i.e. to change the shape of the templates according to the jet energy correct, and then to fit the new templates to the old fit shape, in an attempt to reduce the impact of statistical fluctuations in the data). 
As the latter method produced a larger change in signal yield, it was used for this analysis. As noted for $\gamma$ energy scale, jet energy scale also changes the acceptance rate, changing the unfolding factor, which slightly reduces the uncertainty associated with jet energy scale.

In addition to uncertainties in measurements at CDF, Monte Carlo data sample generation and cross section estimation is highly dependent upon the relative likelihood of a particular parton being the active element in a particular interaction (this is known as the parton distribution function, or pdf, not to be confused with a statistical probability distribution function). The associated uncertainties will therefore be reflected in any Monte Carlo sample or calculation. While these cannot, of course, be measured directly, it is possible to estimate these based on experimental results combined with theoretical calculations.

The likelihood of any particular parton being involved in a given interaction is based on a linear combination of 20 orthogonal eigenvectors, each of which has an uncertainty in its weighting factor. As the 20 orthogonal CTEQ6M[48] eigenvectors are used to weight the Monte Carlo samples, these can be re-weighted in accordance with each of these eigenvectors (twice, $\pm 1 \sigma$, for each eigenvector), and the adjusted Monte Carlo samples can be used as templates for the neural network fit for this analysis. For each re-weighting, the deviation in signal yield was measured, with the larger deviation associated with a given eigenvector taken to be the uncertainty associated with that particular eigenvector. These 20 values were added in quadrature to determine the total uncertainty in yield due to uncertainties in pdf weighting, providing an uncertainty in signal yield of 16.1 events. The acceptance rate was not deemed to depend sufficiently on pdf weighting to merit a calculation of the impact on unfolding factor. 


\subsection{Uncertainties in unfolding factor}

In addition to changes in acceptance rate based on energy scale, there are established cases where Monte Carlo has been shown not to reflect data observed at CDF. As noted by previous work [49] [50], current Monte Carlo $b$ tag efficiency slightly exceeds that for data, so it is necessary to scale the Monte Carlo by $0.958 \pm$ 0.05 (which is squared to account for the second $b$ tag) in order to simulate this effect. Studies of the $Z \rightarrow e^{+} e^{-}$efficiency have also shown a difference in modeled photon acceptance, which varies as a function of the number of vertices present [51]. These uncertainties were applied to the reconstructed Monte Carlo samples, and the change in the resulting unfolding factor was calculated. While uncertainties in energy scale also impact the unfolding factor, since these are coupled to signal yield uncertainties, the coupled uncertainties are the values listed in Table 10.2. 


\section{CHAPTER 10 \\ CROSS SECTION MEASUREMENT AND UNCERTAINTY}

Based on Pythia Monte Carlo calculations, this analysis predicts a cross section of $\sigma=0.35 \mathrm{pb}$ for events which pass all kinematic cuts. This includes a correction (k-factor) of 1.41 since the Pythia calculation included only leading order signal events, and must be adjusted to account for higher-order events. This value is obtained from the predicted value for hadronic cross section described in [52] (linear term in fit in source appears to have sign reversed, based on plot shown, which is corrected here).

The calculated cross section for $p \bar{p} \rightarrow Z \gamma$ with $Z \rightarrow b \bar{b}$ with the kinematic cuts for this analysis $\left(50<m_{j j}<110 \mathrm{GeV}, m_{\gamma j j}>80 \mathrm{GeV}\right.$, leading jet $E_{T}>30$ $\mathrm{GeV}$, secondary jet $E_{T}>20 \mathrm{GeV}, E_{T}^{\gamma}>15 \mathrm{GeV}, E_{T}^{i s o}<1 \mathrm{GeV}, \Delta R_{j \gamma}>0.7$, and $\Delta R_{j j}>1.5$ ) is $\sigma=0.36 \pm 0.16$ (stat.) \pm 0.11 (sys.) pb, which is consistent with the predicted value. Statistical and systematic uncertainties are summarized in Tables 10.1 and 10.2 .

Table 10.1: Statistical uncertainties in this analysis and the resulting impact on cross section.

\begin{tabular}{lc}
\hline Source of Uncertainty & Impact of Uncertainty on Cross Section $(\mathrm{pb})$ \\
\hline Neural Network Fit & 0.161 \\
Fake $\gamma$ Rate & 0.002 \\
Fake $b$ Rate & 0.006 \\
Unfolding factor & 0.004 \\
\hline Total Statistical Uncertainty & 0.161 \\
\hline
\end{tabular}


Table 10.2: Systematic uncertainties in this analysis and their impact upon our cross section measurement

\begin{tabular}{lc}
\hline Source of Uncertainty & Impact of Uncertainty on Cross Section $(\mathrm{pb})$ \\
\hline Jet Energy Scale & 0.075 \\
Fake $b$ Sideband & 0.049 \\
PDF Weighting & 0.031 \\
Luminosity & 0.022 \\
Fake $\gamma$ Sideband & 0.011 \\
$\gamma$ Energy Scale & 0.003 \\
Unfolding $\gamma$ efficiency & 0.008 \\
Unfolding $b$ efficiency & 0.027 \\
SVT trigger rate & 0.032 \\
SVT trigger Energy-dependence & 0.022 \\
\hline Total Systematic Uncertainty & 0.109 \\
\hline
\end{tabular}




\section{CHAPTER 11}

\section{CONCLUSIONS}

This analysis measures the cross section associated with $Z \gamma$ production where $Z$ decays into $b \bar{b}$ at the CDF detector from the Tevatron Run II dataset to be $0.36 \pm 0.16$ (stat.) \pm 0.11 (sys.) pb, consistent with Monte Carlo predictions of 0.35 pb. This analysis is limited by large statistical uncertainty in the neural network fit, and is also rather sensitive to the jet energy scale. With some further optimization, this analysis could be extended to a search for exotics, such as supersymmetry[53] or technicolor[54]. Since the Higgs group at CDF developed a more sophisticated method of identifying $b$ jets, future analyses might be able to improve upon this result using that method. A more sophisticated neural network (likely reliant on next-to-leading-order signal Monte Carlo, which was not available for this analysis) might be able to improve upon the current result. This analysis did not identify any clear departure from measurements of $Z \gamma$ production in leptonic channels, and is not sensitive enough to greatly limit the bounds on such deviations. 


\section{APPENDIX \\ SILICON DETECTOR EXPERIENCE}

\section{A.1 Silicon Detector Technical Details}

During CDF Run II, three silicon sub-detectors were used to track outgoing charged particles. Together, these three sub-detectors comprised the silicon detector system, and were responsible for the highest-sensitivity tracking near the interaction point. Although these were used together in event reconstruction and vertex identification, as each had its own control and readout electronics, the system is generally described as consisting of three sub-detectors.

The primary silicon tracker is known historically as the Silicon Vertex detector, or SVX (SVX II for the Run II version of this detector), and consists of 6 doublesided layers of silicon strips, set up to identify interaction point(s) present during one beam crossing by observing the paths of outgoing charged particles. At $90 \mathrm{~cm}$ in length and extending $10 \mathrm{~cm}$ from the beam pipe, the active region of the SVX II detector provides tracking coverage out to $\eta$ of 2.7 (as seen in Figure A.2[31].

To improve impact parameter resolution, an additional sub-detector, known as Layer 00, consisting of one single-sided layer of silicon tracking strips, was added for the Run II design and was mounted directly on the beam pipe. This exposed the electronics of this section to additional radiation (and the occasional errant particle), requiring that Layer 00 implement a less sophistical design than feasible for the SVX II detector.

Outside of the SVX detector, additional silicon tracking was provided by Intermediate Silicon Layers (ISL), which were located between the SVX region and the Central Outer Tracker (a gas chamber tracker which provided the tracking in the region from $40 \mathrm{~cm}$ to about $140 \mathrm{~cm}$ from the beam pipe, but whose performance deteriorated $\eta>1$ for geometric reasons). The ISL detector was separated into 
five distinct bulkheads, with one central (for $\eta<1.0$ ), and two more on each of the sides (to provide more tracking information in the region where $1.0<\eta<2.0$ ). This coverage in $\eta$ is shown graphically in Figure A.1. Each bulkhead consists of one double-sided silicon layer, but the greater physical space available allows for better resolution than would a single layer of the SVX detector[31].

At the time SVX II was designed, silicon pixel detectors (used for the CMS detector at the LHC) were not considered to be feasible at CDF[26]. A careful analysis of Run II luminosity data for the Tevatron will illustrate that the D0 detector was able to start taking data slightly earlier in each store. This is because the CDF detector could not apply the high voltage to the Layer 00 sub-detector while the beam was being collimated for physics data-taking (a process known internally as scraping), which meant that the CDF detector consistently would become active slightly later than the D0 detector. As this time featured the highest number of interactions, it had a larger impact on integrated luminosity incorporated into the dataset.

\section{A.2 Experience with CDF Silicon Detector}

Due to radiation levels higher than those received by the rest of the detector, as well as the readout demands placed on the electronics, the silicon detector system at CDF typically experienced a greater rate of voltage trips and other electronics failures than the rest of the detector. I had the opportunity to provide on-call technical support for the silicon detector at CDF during some of the last year of its operation.

\section{A.3 Technical challenges in detector construction}

There were a few compromises in design due to problems during installation and early in operation. The first of which was that some of the liquid cooling lines 


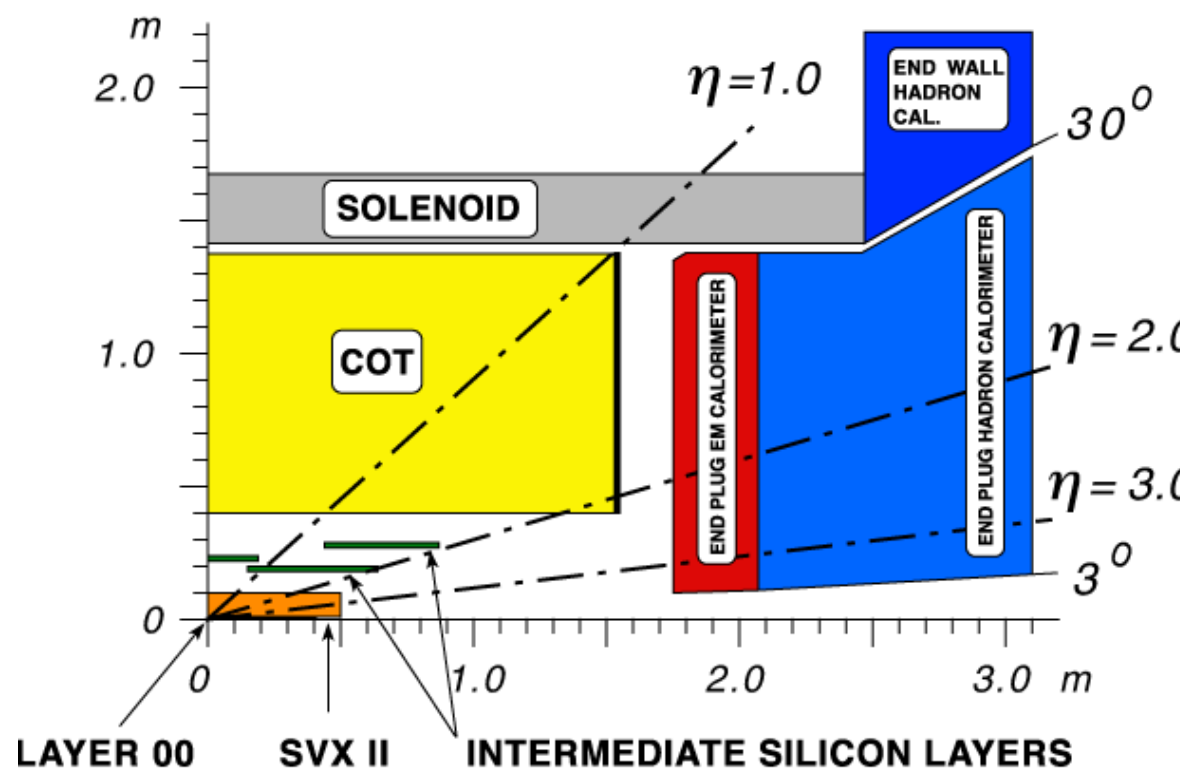

Figure A.1: A horizontally compressed view of the CDF detector, showing $\eta$ coverage.[26]

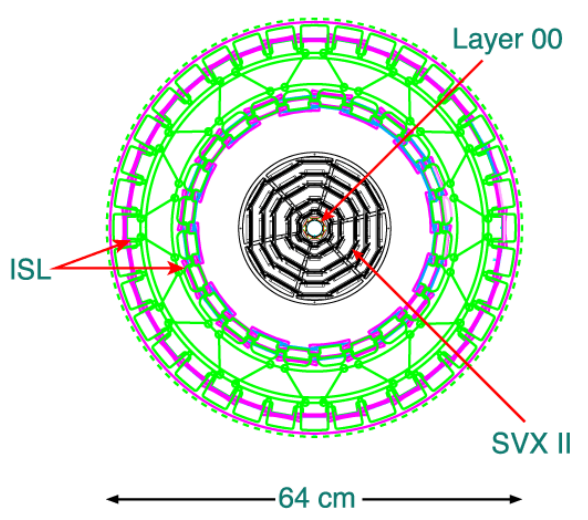

(a) End view of Silicon Detector

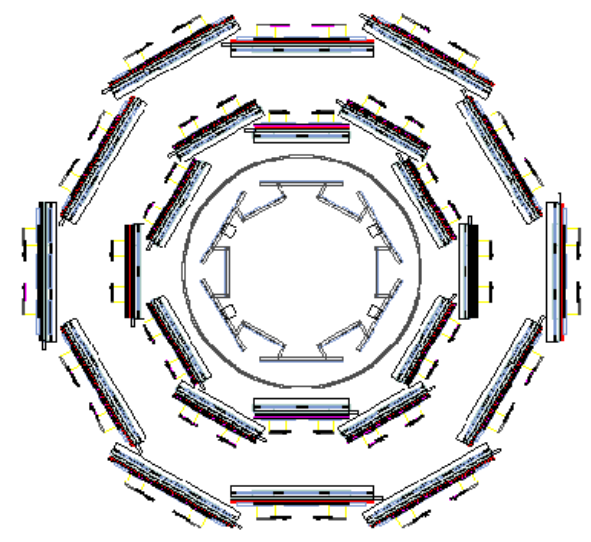

(b) End view of SVX and L00 Detectors

Figure A.2: CDF Run II Silicon detector, end view.[26] 
(for the electronics in the detector) were blocked by epoxy used to connect the joints during construction. While most of these were able to be opened by judicious use of a cutting laser, some remained out of commission for the duration of the experiment[31].

A more unique problem was posed by the wire bonds used to connect the readout electronics to the silicon strips. When an event was flagged by the event triggers as requiring readout, an electric signal would be sent through the wire bond. As the entire tracking region is inside of the large current magnetic solenoid, the wire bond experience a force, in accordance with basic electrodynamics. If a sufficiently large number of events are flagged consecutively, this force is experienced periodically. This causes the wire bond to vibrate, even to the point where (for some of the bonds) the bond breaks, cutting off all communication with that particular readout device[31].

As reworking the electronics was infeasible (due to the nature of the detector and the sensitivity of the whole design), a software solution was developed[31]. The mechanism was simple: an additional circuit board would monitor the number of consecutive events which sent signals through these wire bonds, and send an interrupt when more than 10 signals in a row were sent within one microsecond of the previous signal. This happened an average of 10 times per week[31]. If this started to happen more frequently, there would be discussions about how the trigger might be adjusted to be more friendly to the silicon.

As the experiment progressed over the years, electronic monitoring and recovery improved for the silicon detector. A standard procedure was developed for notifying the shift crew (those individuals responsible for keeping the detector running continuously) when the current for a particular electronic component was out of acceptable limits. A quick flowchart helped the shift crew determine whether to 
call in an expert, or simply to make a short-term note, so that the problem could be addressed when I (or another silicon expert) had time to look at it. The power to each component of the silicon detector was monitored remotely (my first call as pager carrier was when the software that did this froze). Also, various software methods were developed to keep the intervention needed to a minimum. One such program was called "Silicon Auto-Recovery" and was able to turn on individual lines after a high voltage trip (or similar problem)[31]. Another advancement allowed for power supply crates to be rebooted (in case something went wrong there) from the control room, rather than the floor below. Further development allowed for this to be attempted automatically if communication was lost with a crate. Unfortunately, even with these improvements, sometimes there would be an issue that required a more complex solution. Which is when, if I was carrying the pager, I would receive a page. Another development was a way of interacting with the standard measurements of the Tevatron's behavior to determine if the system was safe for the silicon detector to operate (eventually, this would send the voltage in the silicon detector systems to "standby" without human intervention, if something triggered the monitoring software)[31]. If an electronic component inside the detector itself were to fail, replacement was impossible. However, the power supply controls for individual parts of the silicon detector were housed in crates placed in the corners of the collision hall, but outside of the detector itself. At times, one of these would fail completely and require replacement. Part of my job was to make sure that we had spares available, and to help replace one of these power supplies if needed. For safety reasons, this only happened when there was no beam at all in the Tevatron, in what was known as a "controlled access."

The continual operation of detector did require constant monitoring from the control room. This was done by three people: the Consumer Operator (who checked 
that the data coming from the detector made sense with expectations, at least in aggregate, and for checking the validity of calibrations), the Science Coordinator (who made sure that any problems were documented and addressed, and determined if a given dataset was acceptable for use in analysis), and the ACE (which I will cover in more detail). I spent one shift of 12 weeks as ACE, being in the control room 8 hours at a time, for roughly half the week. As ACE, I was responsible for making sure that the high voltage was operational in all parts of the detector and that the data collection software was operating properly, as well as running the data collection software and performing calibrations. This meant being able to diagnose problems (at least in terms of what system was not behaving properly and the symptoms of what was observed), being able to correct minor problems (documented by the experts for each part of the detector), and knowing which person to ask the Science Coordinator to page for a given problem. Since the Consumer Operator and Science Coordinator worked in one week shifts (eight hours a day), as the ACE, I was considered the expert on what was happening when something was going wrong. Typically, this also meant documenting the problem to identify which component was responsible for downtime. Also, when someone needed to be paged to handle a problem, it was typically my task, as the ACE, to know the control room well enough to be talked through minor fixes. Major (or more complicated) fixes would sometimes require that an individual actually drive to the control room to address the problem. I did this a few times as Silicon pager carrier.

Although the detector was sufficiently established that most potential problems were documented, I was still able to provide some of the necessary technical support to keep the detector in operation, both as ACE and as Silicon Pager Carrier. 


\section{REFERENCES}

[1] S. L. Glashow, "Partial-Symmetries of Weak Interactions", Nuclear Physics, 1961, 22, 579 .

[2] D. J. Gross and F. Wilczek, "Ultraviolet Behavior of Non-Abelian Gauge Theories", Physical Review Letters, 1973, 30, 1343.

[3] S. Weinberg, "A Model of Leptons", Physical Review Letters, 1967, 19, 1264.

[4] P. W. Higgs, "Broken Symmetries and the Masses of Gauge Bosons", Physical Review Letters, 1964, 13, 508.

[5] U. Baur and E. L. Berger, "Probing the Weak Boson Sector in $Z \gamma$ Production at Hadron Colliders", Physical Review D, 1993, 47, 4889.

[6] F. Abe et al. (CDF Collaboration), "Limits on Z-Photon Couplings from $p-\bar{p}$ Interactions at $\sqrt{s}=1.8$ TeV", Physical Review Letters, 1995, 74, 1941.

[7] S. Abachi et al. (D0 Collaboration), "Limits on the Anomalous $Z Z \gamma$ and $Z \gamma \gamma$ Couplings in $p \bar{p}$ Collisions at $\sqrt{s}=1.8$ TeV", Physical Review Letters, 1995, 75, 1028.

[8] V. Brigljevic, "First Measurements of Diboson Processes at the LHC", CMS Note, 2013, March 7, 2013.

[9] J. Freeman et al., "Anomalous Coupling Limits from $Z+\gamma$ Production Combining Electronic and Muonic Channels in 5/fb", CDF Note 10043 2010, January $12,2010$.

[10] V. M. Abazov et al. (D0 Collaboration), "Measurement of the $Z \gamma \rightarrow \nu \bar{\nu} \gamma$ Production Cross Section and Limits on Anomalous $Z Z \gamma$ and $Z \gamma \gamma$ Couplings in $p \bar{p}$ Collisions at $\sqrt{s}=1.96$ TeV", Physical Review Letters, 2009, 102, 201802.

[11] S. Weinberg, "Baryon- and Lepton-Nonconserving Processes", Physical Review Letters, 1979, 43, 1566.

[12] G. "t Hooft, "Renormalization of Massless Yang-Mills Fields", Nuclear Physics, 1971, B33, 173.

[13] M. Gell-Mann, "A Schematic Model of Baryons and Mesons", Physics Letters, 1964, 8, 214. 
[14] Fermilab National Acceleration Laboratory. Science at Fermilab. http://www.fnal.gov/pub/science/index.html (accessed March 1, 2013).

[15] W. M. Yao et al., "Review of Particle Physics", Journal of Physics, G 33, 1 (2006).

[16] J. D. Bjorken and E. A. Paschos, "Inelatic Electron-Proton and $\gamma$-Proton Scattering and the Structure of the Nucleon", Physical Review, 1969, 185, 1975.

[17] D. J. Griffiths, Introduction to Elementary Particles, 2nd ed.; John Wiley and Sons: New York City, 1987.

[18] P. D. B. Collins and N. A. Speirs, "Composite Weak Bosons and Their Decays", Journal of Physics G: Nuclear Physics, 1986, 12, 1115.

[19] A. Denner, et al., "Standard Model Higgs-Boson Branching Ratios with Uncertainties", European Physical Journal C, 2011, 71, 1753.

[20] U. Baur, et al., "Report of the Working Group on Weak Boson Production" Report of the Proceedings of the Workshop "QCD and Weak Boson Physics in Run II". [online] 2000 http://arxiv.org/abs/hep-ph/0005226 (updated November 3, 2000).

[21] E. O. Lawrence, "Method and Apparatus for the Acceleration of Ions", U.S. Patent 1948384, February 20, 1934.

[22] E. McMillan, "Synchron-Cyclotron", U.S. Patent 2615129, October 21, 1952.

[23] G. Aarons et al., International Linear Collider Reference Design Report, [online] 2007

http://arxiv.org/abs/0709.1893 (Updated September 12, 2007).

[24] R. Lipton, Muon Collider: Plans, Progress and Challenges, [online] 2012 http://arxiv.org/abs/1204.3538 (Updated April 16, 2012).

[25] V. Khachatryan et al. (CMS Collaboration), "Transverse-momentum and Pseudorapidity Distributions of Charged Hadrons in $p p$ Collisions at $\sqrt{s}=0.9$ and 2.36 TeV", Journal of High Energy Physics, 2010, 2010:41.

[26] F. Abe et al. (CDF II Collaboration), "The CDF II Detector Technical Design Report", FERMILAB-Pub-96 390-E (1996).

[27] D. Acosta et al. (CDF II Collaboration), "Measurement of the $t \bar{t}$ Production Cross Section in $p \bar{p}$ Collisions at $\sqrt{s}=1.96 \mathrm{TeV}$ Using Lepton + Jets Events with Secondary Vertex b-tagging", Physical Review D, 2005, 71, 052003. 
[28] V. M. Abazov et al. (D0 Collaboration), "The Upgraded D0 Detector", Nuclear Instruments and Methods in Physics Research, 2005, A565, 463.

[29] F. Abe et al. (CDF Collaboration), "Observation of Top Quark Production in $p \bar{p}$ Collisions with the Collider Detector at Fermilab". Physical Review Letters, 1995, 74, 2626-2631.

[30] J. D. Cockroft and E. T. S. Walton, "Experiments with High Velocity Positive Ions", Proceedings of the Royal Society of London, 1932, A137, 229.

[31] T. Aaltonen et al. (CDF II Collaboration), "Operational Experience, Improvements, and Performance of the CDF Run II Silicon Vertex Detector", Nuclear Instruments and Methods in Physics Research, 2013, A729, 153-181.

[32] J. Adelman et al. (CDF II Collaboration), "The Silicon Vertex Trigger Upgrade at CDF", Nuclear Instruments and Methods in Physics Research, 2007, A572, 361.

[33] T. Aaltonen et al. (CDF II Collaboration), "Search for Hadronic Decays of W and $\mathrm{Z}$ bosons in photon events in p p-bar collisions at $\mathrm{s}^{* *}(1 / 2)=1.96-\mathrm{TeV}$," Physical Review D, 80, 052011 (2009) [arXiv:0803.4264 [hep-ex]].

[34] S. Jaykin, Neural Networks: A Comprehensive Foundation; Prentice Hall: New Jersey, 1999.

[35] G. Stimpfl-Abele and P. Yepes, "Higgs Search and Neural-Net Analysis", Computer Physics Communications, 1993, 78, 1.

[36] J. Ray et al., "Training a Multivariate Photon ID Against Jets", CDF Note 10282, 2010, September 6, 2010.

[37] CERN, "ROOT: A Data Analysis Framework", http://root.cern.ch/drupal (updated June 26, 2013).

[38] CERN, " RooFit:ROOT", http://root.cern.ch/drupal/content/roofit (updated June 26, 2013).

[39] F. Maltoni and T. Stelzer, "MadEvent: Automatic Event Generation with MadGraph", Journal of High Energy Physics 02 (2003) 027.

[40] T. Sjostrand et al., "High-energy-physics Event Generation with Pythia 6.1", Comput. Phys. Commun. 135, 238 (2001).

[41] S. Agostinelli et al. (GEANT Collaboration), "GEANT4-A Simulation Toolkit", Nuclear Instruments and Methods in Physics Research, 2003, A506, 250. 
[42] J. Alwall et al., "Comparative Study of Various Algorithms for the Merging of Parton Showers and Matrix Elements in Hadronic Collisions", European Physics Journal, 2008, C53, 473.

[43] A. Bhatti et al., "Determination of the Jet Energy Scale at the Collider Detector at Fermilab", Nuclear Instruments and Methods in Physics Research, 2006, A566, 375.

[44] Collider Detector at Fermilab, "CDF High $p_{T}$ B-Tag Group", http://www-cdf.fnal.gov/internal/physics/top/RunIIBtag/bTag.html (updated March 6, 2012).

[45] J. Freeman et al., "Introduction to HOBIT, a $b$-Jet Identification Tagger at the CDF Experiment Optimized for Light Higgs Boson Searches", CDF Note 10803, 2012, February 27, 2012.

[46] R. McNulty and T. Shears, "Photon + b jet production at CDF", CDF Note 8329, 2006, June 19, 2006.

[47] S. Yu et al., "Studies of Electron and Photon Energy Scales", CDF Note 9591, 2008, December 9, 2008.

[48] J. Pumplin et al., "New Generation of Parton Distribution Functions with Uncertainties from Global QCD Analysis" Journal of High Energy Physics 0207:012(2002), hep-ph/0201195.

[49] V. Giakoumopoulou and J. Freeman, "Summer 2010 SecVtx Scale Factors Calculated Using the Electron Method through Period 28", CDF Note 10178, 2010 June 7, 2010.

[50] S. Sabik and P. Savard, "Track Reconstruction Efficiency in Jets", CDF Note 6894, 2004, February 23, 2004.

[51] K. Bland et al., "Search for a SM and Fermiophobic Higgs Boson in the Diphoton Final State", CDF Note 10724, 2012, January 14, 2012.

[52] A. Bocci, "Theoretical Prediction of $W \gamma$ and $Z \gamma$ Production in the Hadronic Channel", CDF Note 7664, 2005, May 23, 2005.

[53] R. Haag, J. T. Lopuszanski, and M. Sohnius, "All Possible Generators of Supersymmetries of the S-Matrix", Nuclear Physics B, 1975, B88, 257.

[54] R. Jackiw, and K. Johnson, "Dynamical Model of Spontaneously Broken Gauge Symmetries", Physical Review D, 1973, 8, 2386. 